\title{
DISCOVERY OF THE YOUNG L DWARF WISE J174102.78-464225.5
}

\author{
Adam C. Schneider ${ }^{1}$, Michael C. Cushing ${ }^{1}$, J. Davy Kirkpatrick $^{2}$, Gregory N. Mace $^{2,3}$, Christopher R. Gelino $^{2,4}$, \\ Jacqueline K. Faherty ${ }^{5}$, Sergio Fajardo-Acosta ${ }^{2}$, and Scott S. Sheppard ${ }^{6}$ \\ ${ }^{1}$ Department of Physics and Astronomy, University of Toledo, 2801 West Bancroft Street, Toledo, OH 43606, USA; Adam.Schneider@Utoledo.edu \\ ${ }^{2}$ Infrared Processing and Analysis Center, MS 100-22, California Institute of Technology, Pasadena, CA 91125, USA \\ ${ }^{3}$ Department of Physics and Astronomy, UCLA, 430 Portola Plaza, Box 951547, Los Angeles, CA 90095-1547, USA \\ ${ }^{4}$ NASA Exoplanet Science Institute, Mail Code 100-22, California Institute of Technology, 770 South Wilson Avenue, Pasadena, CA 91125 , USA \\ ${ }^{5}$ Department of Astronomy, University of Chile, Camino El Observatorio 1515, Casilla 36-D, Santiago, Chile \\ ${ }^{6}$ Department of Terrestrial Magnetism, Carnegie Institution of Washington, 5241 Broad Branch Road NW, Washington, DC 20015, USA \\ Received 2013 October 18; accepted 2013 November 22; published 2014 January 9
}

\begin{abstract}
We report the discovery of the L dwarf WISE J174102.78-464225.5, which was discovered as part of a search for nearby L dwarfs using the Wide-field Infrared Survey Explorer (WISE). The distinct triangular peak of the $H$-band portion of its near-infrared spectrum and its red near-infrared colors $\left(J-K_{S}=2.35 \pm 0.08 \mathrm{mag}\right)$ are indicative of a young age. Via comparison to spectral standards and other red $\mathrm{L}$ dwarfs, we estimate a near-infrared spectral type of $\mathrm{L} 7 \pm 2$ (pec). From a comparison to spectral and low-mass evolutionary models, we determine self-consistent effective temperature, $\log g$, age, and mass values of $1450 \pm 100 \mathrm{~K}, 4.0 \pm 0.25\left(\mathrm{~cm} \mathrm{~s}^{-2}\right), 10-100 \mathrm{Myr}$, and 4-21 $M_{\mathrm{Jup}}$, respectively. With an estimated distance of $10-30 \mathrm{pc}$, we explore the possibility that WISE J174102.78-464225.5 belongs to one of the young nearby moving groups via a kinematic analysis and we find potential membership in the $\beta$ Pictoris or $\mathrm{AB}$ Doradus associations. A trigonometric parallax measurement and a precise radial velocity can help to secure its membership in either of these groups.
\end{abstract}

Key words: brown dwarfs - stars: individual (WISE J174102.78-464225.5) - stars: low-mass

Online-only material: color figures

\section{INTRODUCTION}

The Wide-field Infrared Survey Explorer (WISE) has heralded in an unprecedented era of substellar discovery. WISE surveyed the entire sky at $3.4,4.6,12$, and $22 \mu \mathrm{m}(W 1, W 2, W 3$, and $W 4$ bands), with channels $W 1$ and $W 2$ designed specifically to be sensitive to cold brown dwarfs with effective temperatures $\left(T_{\text {eff }}\right)$ less than $1400 \mathrm{~K}$ (Wright et al. 2010). With the substantial sample of substellar objects discovered by WISE (e.g., Kirkpatrick et al. 2011; Mace et al. 2013; Thompson et al. 2013), we can now perform individual in-depth studies to probe the evolution of such objects. It is critical when investigating the evolution of substellar objects to identify sources at various ages in order to construct a complete evolutionary sequence. The majority of $\mathrm{L}$ and $\mathrm{T}$ dwarfs discovered to date are old (age $>1 \mathrm{Gyr}$ ), consistent with a field population (Dahn et al. 2002; Schmidt et al. 2007; Faherty et al. 2009). Young brown dwarfs are rarer, but have distinctive characteristics that distinguish them from their older counterparts. Since young dwarfs are still contracting to their final radii, their surface gravities are lower, which can produce noticeable effects in the optical and near-infrared spectra of substellar objects (e.g., Kirkpatrick et al. 2006; Cruz et al. 2009; Bonnefoy et al. 2013; Allers \& Liu 2013). Consequently, many young substellar objects have been identified by their low surface gravity spectral features, such as enhanced VO bands, narrow or weak alkali lines, a triangular $H$-band spectral shape, and red near-infrared colors (Cruz et al. 2007, 2009; Kirkpatrick et al. 2008, 2010; Faherty et al. 2012; Thompson et al. 2013). Studying these young field $\mathrm{L}$ and $\mathrm{T}$ dwarfs will help to constrain and improve substellar evolution models, while additional examples of young, late-type substellar objects can help to fill in the evolutionary gaps of these astronomical bodies. These models will become increasingly important in interpreting results from the next generation of extreme adaptive optics imagers (i.e., the Gemini Planet Imager, Project 1640, the Spectro-Polarimetric High-contrast Exoplanet Research, and the Coronagraphic High Angular Resolution Imaging Spectrograph) as they attempt to image young exoplanets directly, since the determination of an accurate age can significantly affect the mass estimates of brown dwarfs and exoplanets (see, for example, the discussion of HR $8799 \mathrm{~b}, \mathrm{c}, \mathrm{d}$, and e in Oppenheimer et al. 2013).

Here we report the discovery of the young, late-type object WISE J174102.78-464225.5 (henceforth WISE 1741-4642), discovered as part of a search for bright $\mathrm{L}$ and $\mathrm{T}$ type objects using WISE data. We summarize the identification of WISE 1741-4642 and our subsequent observations in Section 2. In Section 3 we discuss the spectral and physical characteristics of WISE 1741-4642, and provide a kinematic analysis where we evaluate potential young moving group membership for this object.

\section{IDENTIFICATION AND OBSERVATIONS}

\subsection{Identification}

WISE 1741-4642 was discovered as part of a search for bright $\mathrm{L}$ and $\mathrm{T}$ dwarfs. A complete description of the selection criteria is outlined in Section 2.1 of Thompson et al. (2013). To summarize, candidates are required to have a $W 1-W 2$ color $>0.4$ and a $W 2$ signal-to-noise ratio $>20$. In an attempt to exclude extragalactic objects, candidates are also required to have $W 1-W 2>0.96(W 2-W 3)-0.96$. Candidates are required to have no matching Two Micron All Sky Survey (2MASS) or USNO source within $3^{\prime \prime}$ to ensure moderate to high proper motions. Lastly, candidates are required to not be flagged as a solar system object. This search has resulted in the detection of $55 \mathrm{~L}$ and $\mathrm{T}$ dwarfs, previously discussed in Kirkpatrick et al. (2011), Mace et al. (2013), and Thompson et al. (2013). 


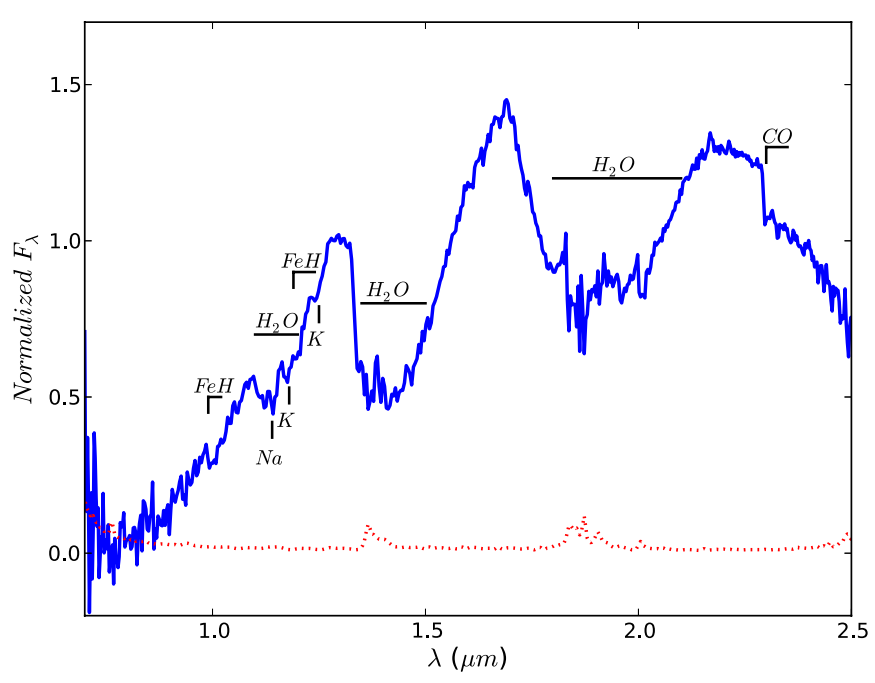

Figure 1. SpeX near-infrared spectrum of WISE 1741-4642. Uncertainties are shown with a red dotted line. Major atomic and molecular features identifiable at low resolution have been labeled.

(A color version of this figure is available in the online journal.)

\subsection{Observations}

\subsubsection{IRTF/SpeX}

We observed WISE 1741-4642 on the night of 2012 July 19 UT employing the medium-resolution infrared spectrograph SpeX (Rayner et al. 2003) at the 3 m NASA Infrared Telescope Facility (IRTF) on Mauna Kea. Observations were conducted in prism mode with a 0 '.5 wide slit, which achieves a resolving power $(\lambda / \Delta \lambda)$ of $\sim 150$ over the range $0.8-2.5 \mu \mathrm{m}$. A series of ten $120 \mathrm{~s}$ exposures were obtained at two different nod positions along the $15^{\prime \prime}$ long slit, which was oriented to the parallactic angle. The A0V star HD 161706 was observed at a similar airmass as WISE 1741-4642 for telluric correction and flux calibration. Calibration spectra of internal flat field and argon arc lamps were obtained for flat fielding and wavelength calibration. The data were reduced with SpeXtool, the IDL-based data reduction package for SpeX (Cushing et al. 2004), which first corrected raw frames for non-linearity then pair subtracted and flat fielded. The spectra were then optimally extracted (e.g., Horne 1986) and wavelength calibrated using argon lamp exposures. Finally, multiple spectra were averaged together, corrected for telluric absorption, and flux calibrated (Vacca et al. 2003). The spectrum was absolutely flux calibrated using 2MASS magnitudes and the procedure outlined in Cushing et al. (2005).

\subsubsection{Magellan/FIRE}

WISE 1741-4642 was observed with the Folded-port Infrared Echellette (FIRE; Simcoe et al. 2013) on the Magellan Baade Telescope on 2013 August 9 and 2013 August $10 \mathrm{UT}$ in cross-dispersed echelle mode $(\lambda / \Delta \lambda \approx 6000)$ from $0.65-2.55 \mu \mathrm{m}$. Four $500 \mathrm{~s}$ exposures were taken each night in an ABBA dither pattern. The A0V stars HIP $84305(V=8.41)$ and HIP $89666(V=8.65)$ were also observed for telluric correction purposes. We also obtained flat-field and arc lamp (ThAr) exposures for pixel response and wavelength calibration. Data were reduced using the FIREHOSE package, which employs modified routines from the SpeXtool data reduction package (Vacca et al. 2003; Cushing et al. 2004).

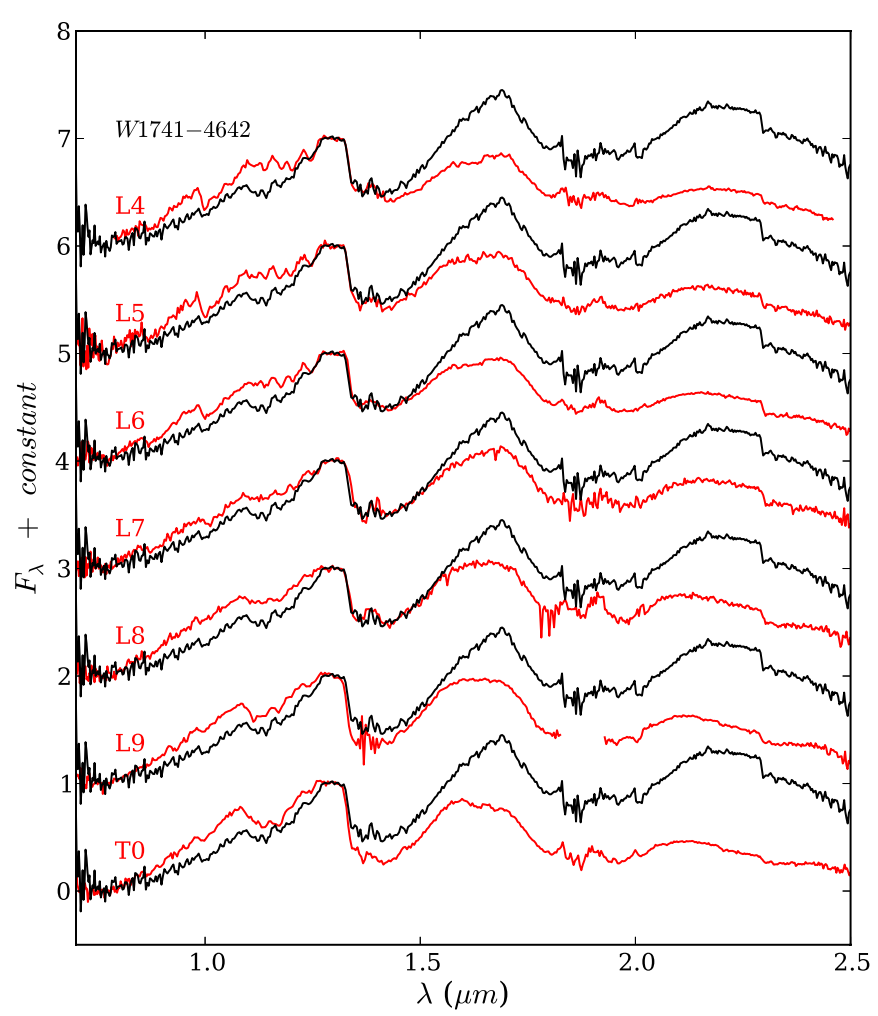

Figure 2. Near-infrared spectrum of WISE 1741-4642 (black) compared with spectral standards (L4 through T0; red) from the Spex Prism Spectral Library. Each spectrum is normalized by the mean flux from 1.27 to $1.32 \mu \mathrm{m}$. The spectra are separated along the vertical axis by a constant of 1.0 for clarity. The spectral standards used for this comparison are as follows: 2MASS J21580457-1550098 (L4; Kirkpatrick et al. 2010), SDSS J083506.16+195304.4 (L5; Chiu et al. 2006), 2MASSI J1010148-040649 (L6; Reid et al. 2006), 2MASSI J0103320+193536 (L7; Cruz et al. 2004), 2MASSW J1632291+190441 (L8; Burgasser 2007a), DENIS-P J0255-4700 (L9; Burgasser et al. 2006), and SDSS J120747.17+024424.8 (T0; Looper et al. 2007). (A color version of this figure is available in the online journal.)

\section{DISCUSSION}

\subsection{Comparison to Spectral Standards}

The SpeX near-infrared spectrum is presented in Figure 1, with prominent atomic and molecular lines labeled. We first attempted to determine the spectral type of WISE 1741-4642 by comparing the $J$-band spectral morphology with that of the near-infrared spectral standards (from Kirkpatrick et al. 2010, obtained from the Spex Prism Spectral Library ${ }^{7}$ ). A comparison of WISE 1741-4642 to several spectral standards is shown in Figure 2. The figure makes clear that WISE 1741-4642 is redder than normal L dwarfs, and a single well-fitting spectral type is not easily determined by direct comparison (see Mace et al. 2013 for an in depth discussion on the difficulties encountered when comparing red dwarfs to spectral standards). We conservatively estimate the spectral type of WISE $1741-4642$ to be L7 \pm 2 from the comparison to $\mathrm{L}$ and $\mathrm{T}$ dwarf spectral standards based on $\mathrm{H}_{2} \mathrm{O}$ and $\mathrm{FeH}$ band strengths.

We also investigated the near-infrared spectral type indices examined by Allers \& Liu (2013). Most of these indices are degenerate for spectral types later than L5. Because the byeye comparisons suggest a spectral type later than L5, only the $\mathrm{H}_{2} \mathrm{OD}$ index, originally defined by McLean et al. (2003), is useful. We calculated an $\mathrm{H}_{2} \mathrm{OD}$ index of 0.78 , which, utilizing

\footnotetext{
7 http://pono.ucsd.edu/ adam/browndwarfs/spexprism/library.html
} 


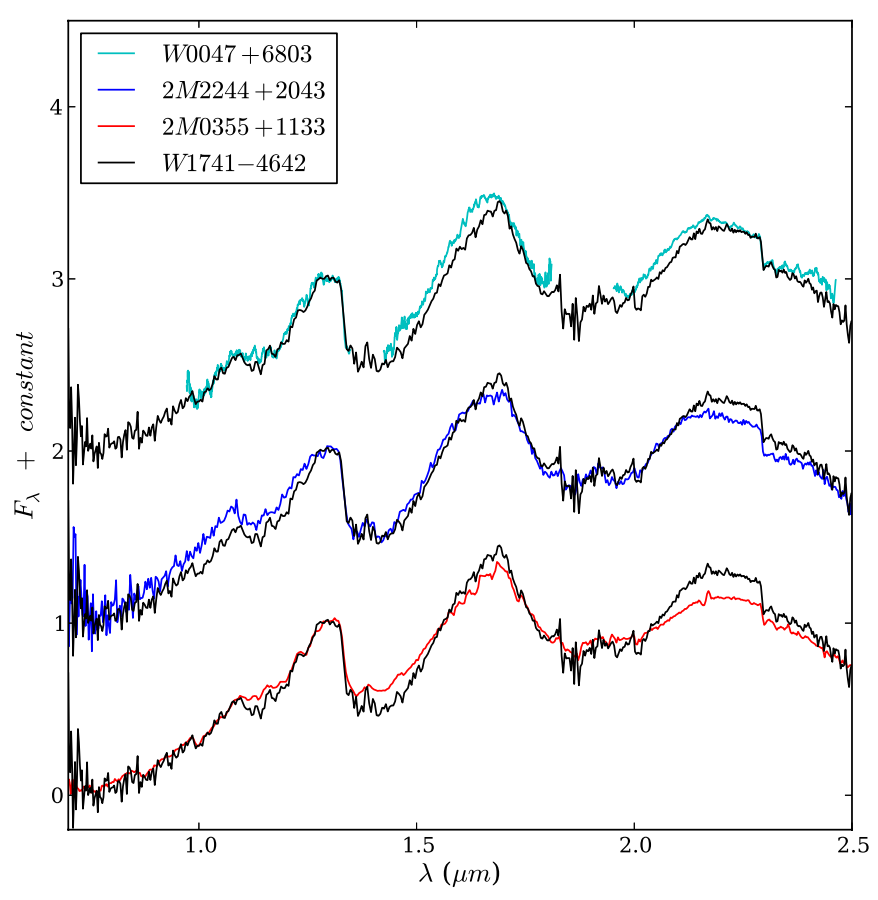

Figure 3. Near-infrared spectrum of WISE 1741-4642 (black) compared with the unusually red L dwarfs W0047+6803 $\left(\mathrm{SpT}_{\mathrm{IR}}=\mathrm{L} 7.5\right.$ (pec); Gizis et al. 2012), 2M2244+2043 (SpT $\mathrm{SR}_{\mathrm{IR}}=\mathrm{L} 7.5$ (pec); Kirkpatrick et al. 2008), and $2 \mathrm{M} 0355+1133\left(\mathrm{SpT}_{\mathrm{opt}}=\mathrm{L} 5 \gamma\right.$; Faherty et al. 2013). Each spectrum is normalized by the mean flux from 1.27 to $1.32 \mu \mathrm{m}$. While the spectra match better than the spectral standards in Figure 2, WISE 1741-4642 is redder than both $2 \mathrm{M} 0355+1133$ and $2 \mathrm{M} 2244+2043$ and more sharply peaked at $\mathrm{H}$ than 2M2244+2043.

(A color version of this figure is available in the online journal.)

the polynomial fits in Table 3 of Allers \& Liu (2013), gives a spectral type of $\sim$ L5.5.

\subsection{Comparison to Red L Dwarfs}

The spectrum of WISE 1741-4642 was compared to other unusually red late-type $\mathrm{L}$ dwarfs to aid in constraining its spectral type. Figure 3 shows the near-infrared SpeX spectrum of WISE 1741-4642 compared with the red L dwarf 2MASSW J224431.67+204343.3 (2M2244+2043; SpT (NIR) $=$ L7.5 (pec), Kirkpatrick et al. 2008; SpT = L6 (VL-G), Allers \& Liu 2013), the young L dwarf 2MASS J035523.27+113343.7 (2M0355+1133; SpT (opt) $=$ L5 $\gamma$, Faherty et al. 2013), and the red L dwarf WISEP J004701.06+680352.1 (W0047+6803; SpT (NIR) = L7.5 (pec), Gizis et al. 2012). We derive a nearinfrared spectral type of L7 (pec) for 2M0355+1133 using the method outlined in the Appendix. All three spectra match WISE $1741-4642$ reasonably well, suggesting a spectral type range of L7-L8. While these red L dwarfs are much better comparisons than the L dwarf spectral standards, differences can still be seen. Figure 3 shows that WISE 1741-4642 is both redder than $2 \mathrm{M} 0355+1133$ and $2 \mathrm{M} 2244+2043$ and has a more peaked $H$-band profile than 2M2244+2043. WISE 1741-4642 also shows deeper $\mathrm{H}_{2} \mathrm{O}$ absorption than 2M0355+1133. $\mathrm{W} 0047+6803$ is the best match at $\mathrm{J}$, though it is slightly redder than WISE 1741-4642. A more detailed comparison of individual spectral lines is performed in Section 3.4.2. Combining the estimated spectral type of WISE 1741-4642 from the comparison of spectral standards, the $\mathrm{H}_{2} \mathrm{OD}$ spectral type index, and the comparison to other red L dwarfs, we assign a spectral type of L7 \pm 2 (pec) to WISE 1741-4642.

\subsection{Comparison to Model Spectra}

The "BT-Settl" models from the Phoenix/NextGen group (Allard et al. 2011), using the solar abundances from Asplund et al. (2009), were used to estimate the $T_{\text {eff }}$ and $\log g$ values of WISE 1741-4642. We compared models from 1000 to $2000 \mathrm{~K}$ in steps of $100 \mathrm{~K}$ and $\log g$ values of 3.5 to $5.5\left(\mathrm{~cm} \mathrm{~s}^{-2}\right)$ in steps of 0.5 dex to our measured SpeX spectrum. In order to compare the models to our measured spectrum, each model spectrum was resampled to be uniform in $\ln (\lambda)$ space, smoothed with a Gaussian kernel to a resolving power of 150, and resampled onto the same wavelength grid as the SpeX spectrum. Following Cushing et al. (2008), a goodness of fit parameter is evaluated for each model spectrum. The goodness of fit parameter is defined as

$$
G_{k}=\sum_{i=1}^{n}\left(\frac{f_{i}-C_{k} \mathcal{F}_{k, i}}{\sigma_{i}}\right)^{2},
$$

where $n$ corresponds to each data pixel, $f_{i}$ is the flux density of the data, $\mathcal{F}_{k, i}$ is the flux density of the model $k$, and $\sigma_{i}$ are the errors for each observed flux density. The $C_{k}$ parameter is a multiplicative constant given by

$$
C_{k}=\frac{\sum f_{i} \mathcal{F}_{k, i} / \sigma_{i}^{2}}{\sum \mathcal{F}_{k, i} / \sigma_{i}^{2}}
$$

$C_{k}$ is equivalent to $(R / d)^{2}$, where $R$ is the stellar radius and $d$ is the distance (see Section 3.4.1). We performed a Monte Carlo simulation using our observed spectrum to determine the uncertainty of the best fitting spectrum (e.g., Cushing et al. 2010). We generated 1000 simulated data sets using both the absolute flux calibration uncertainty and the individual flux uncertainties at each wavelength. Each measured flux in the SpeX spectrum is randomly drawn from a single Gaussian distribution centered on the observed flux with a width given by the observed variance. The entire spectrum is also multiplied by a factor randomly drawn from a Gaussian distribution centered at one with a width given by the absolute flux calibration uncertainty.

Several low-gravity spectral models provided the best fits (Figure 4). The best fitting model spectrum was that with $T_{\text {eff }}=1600 \mathrm{~K}$ and a $\log g$ value of 3.5. In Section 3.4.3 we show that a $T_{\text {eff }}$ of $1600 \mathrm{~K}$ and a $\log g$ of 3.5 suggest an unphysical age of $\leqslant 5$ Myr for WISE 1741-4642. Therefore, we selected the next two best fitting spectral models $\left(T_{\text {eff }}=1500 \mathrm{~K}\right.$ and $\log g=4.0 ; T_{\text {eff }}=1400 \mathrm{~K}$ and $\left.\log g=4.0\right)$ for further analysis. The three best fitting models are displayed in Figure 4. We take $T_{\text {eff }}=1450 \pm 100 \mathrm{~K}$ and $\log g=4.0 \pm 0.25\left(\mathrm{~cm} \mathrm{~s}^{-2}\right)$ as our final estimates for these parameters. This temperature is in agreement with temperatures deduced for other $\sim \mathrm{L} 7 \mathrm{dwarfs}$ (Golimowski et al. 2004).

\subsection{Physical Characteristics \\ 3.4.1. Distance}

A byproduct of the model fitting technique is an estimate of the multiplicative constant $C_{k}$ that is equivalent to $(R / d)^{2}$, where $R$ is the radius of the star and $d$ is the distance from Earth. Therefore, a stellar radius, in combination with model spectrum comparisons, can provide a distance estimate. We utilized four sets of low-mass evolutionary models combined with our best fit $T_{\text {eff }}$ and $\log g$ ranges to estimate a stellar radius: the "COND" models (Baraffe et al. 2003), the "DUSTY" models (Chabrier et al. 2000), and the cloudy and cloudless models of Saumon 

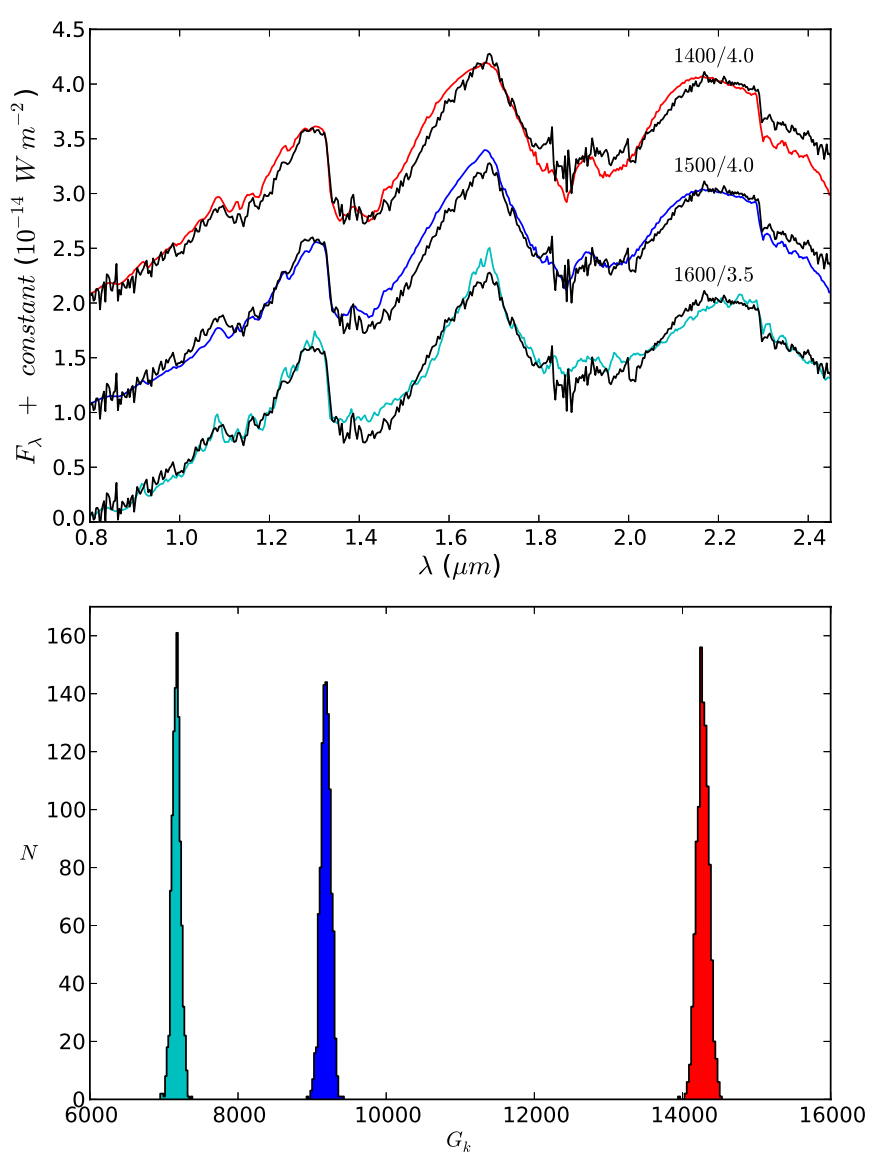

Figure 4. Top: the near-infrared spectrum of WISE 1741-4642 (black) compared with the three best fitting BT-Settl models. Each model spectrum is labeled with its corresponding $T_{\text {eff }}$ and $\log g$ values. Bottom: the distribution of $G_{k}$ values for the three best fitting models. Red: $T_{\text {eff }}=1400 \mathrm{~K}$ and $\log g$ $=4.0$, blue: $T_{\text {eff }}=1500 \mathrm{~K}$ and $\log g=4.0$, and cyan: $T_{\text {eff }}=1600 \mathrm{~K}$ and $\log g=3.5$.

(A color version of this figure is available in the online journal.)

\& Marley (2008). For the "DUSTY" models, dust grains are suspended, while in the "COND" models dust is removed from the photosphere. Similarly, the cloudy and cloudless models of Saumon \& Marley (2008) account for cloud sedimentation, where the sedimentation efficiency parameter $\left(f_{\text {sed }}\right)=2$ for the cloudy models and the cloudless models include no opacity from clouds. We found a radius range of $0.137-0.161 R_{\odot}$ $\left(1.36-1.60 R_{\mathrm{Jup}}\right)$ for the "DUSTY" and "COND" models, and a range of $0.1398-0.1752 R_{\odot}\left(1.39-1.74 R_{\text {Jup }}\right)$ for the Saumon $\&$ Marley (2008) models. This range, in combination with the $C_{k}$ values determined via model fitting, result in a so-called "spectroscopic parallax" (Bowler et al. 2009) of 8-11 pc.

We also estimate photometric distances using the relations of Looper et al. (2008a), Kirkpatrick et al. (2011), and Dupuy \& Liu (2012). For a spectral type range of L5-L9 and the 2MASS and WISE magnitudes given in Table 1, we calculate distance ranges of $12-25 \mathrm{pc}$ and $11-28 \mathrm{pc}$ for the Looper et al. (2008a) and Dupuy \& Liu (2012) relations, respectively. For the WISE W2 relation of Kirkpatrick et al. (2011), we calculated a distance range of 11-14 pc. The distance estimates using the $K_{S}, W 1$, and $W 2$ magnitudes provided the closest matches to the distance derived via spectroscopic parallax (11-20 pc, 11-17 pc, and 10-15 pc for $K_{S}, W 1$, and $W 2$, respectively), which could imply that the magnitudes from the wavelength region between $K_{S}$ and WISE W2 are the least affected by youth.
Table 1

WISE 1741-4642 Properties

\begin{tabular}{|c|c|c|}
\hline Parameter & Value & Ref. \\
\hline \multicolumn{3}{|c|}{ Observed properties } \\
\hline$\alpha(\mathrm{J} 2000)$ & $17: 41: 02.79$ & 1 \\
\hline$\delta(\mathrm{J} 2000)$ & $-46: 42: 25.55$ & 1 \\
\hline$\mu_{\alpha} \cos \delta\left(\operatorname{mas}_{\mathrm{yr}} \mathrm{r}^{-1}\right)$ & $-20.4 \pm 9.2$ & 3 \\
\hline$\mu_{\delta}\left(\operatorname{mas} \mathrm{yr}^{-1}\right)$ & $-343.0 \pm 13.7$ & 3 \\
\hline$v_{\text {rad }}$ & $-5.7 \pm 5.1 \mathrm{~km} \mathrm{~s}^{-1}$ & 3 \\
\hline$J$ (mag) & $15.786 \pm 0.075$ & 1 \\
\hline$H$ (mag) & $14.534 \pm 0.054$ & 1 \\
\hline$K_{S}(\mathrm{mag})$ & $13.438 \pm 0.035$ & 1 \\
\hline$W 1$ (mag) & $12.301 \pm 0.025$ & 2 \\
\hline$W 2$ (mag) & $11.675 \pm 0.023$ & 2 \\
\hline W3 (mag) & $11.432 \pm 0.190$ & 2 \\
\hline W4 (mag) & $<12.301$ & 2 \\
\hline$J-H(\mathrm{mag})$ & $1.25 \pm 0.09$ & 1 \\
\hline$J-K_{S}(\mathrm{mag})$ & $2.35 \pm 0.08$ & 1 \\
\hline$J-W 2(\mathrm{mag})$ & $4.11 \pm 0.08$ & 1,2 \\
\hline$W 1-W 2(\mathrm{mag})$ & $0.63 \pm 0.03$ & 2 \\
\hline \multicolumn{3}{|c|}{ Inferred properties } \\
\hline Spectroscopic distance & $8-11 \mathrm{pc}$ & 3 \\
\hline Photometric distance & $10-30 \mathrm{pc}$ & 3 \\
\hline Spectral type (NIR) & $\mathrm{L} 7 \pm 2(\mathrm{pec})$ & 3 \\
\hline$T_{\text {eff }}$ & $1450 \pm 100 \mathrm{~K}$ & 3 \\
\hline $\log g$ & $4.0 \pm 0.25\left(\mathrm{~cm} \mathrm{~s}^{-2}\right)$ & 3 \\
\hline Mass & $4-21 M_{\text {Jup }}$ & 3 \\
\hline Age & $10-100 \mathrm{Myr}$ & 3 \\
\hline
\end{tabular}

References. (1) 2MASS catalog (Cutri et al. 2003); (2) WISE All-Sky Catalog (Cutri et al. 2012); (3) This paper.

This is further supported by Figure 9 of Faherty et al. (2013), which shows that the absolute flux of $2 \mathrm{M} 0355+1133$ matches the field L5 2M1507-16 well at K, while it is underluminous everywhere blueward of $\sim 2 \mu \mathrm{m}$. Liu et al. (2013) also find that many of their young mid-L dwarfs with measured parallaxes are fainter at $\mathbf{J}$ than field objects of comparable spectral type. This is supported by the results of Faherty et al. (2012), in which they find that $\sim 80 \%$ of objects in their low-gravity and young companion L dwarf sample are underluminous at J. According to Table 10 of Faherty et al. (2012), low-gravity and young companions are, on average, 0.48 mag underluminous at $\mathrm{J}$ and only $0.17 \mathrm{mag}$ underluminous at K. If WISE $1741-4642$ is similarly underluminous at $\mathrm{J}$, then one would expect the distance estimates based on its $J$-band magnitude to be overestimated. We found that the $J$-band photometric distances were the largest of all estimates: $17-28 \mathrm{pc}$ and $16-26 \mathrm{pc}$ for spectral types L5-L9 according to the Dupuy \& Liu (2012) and Looper et al. (2008a) relations, respectively. We conservatively take $10-30 \mathrm{pc}$ as our final distance estimate.

\subsubsection{Empirical Age}

The near-infrared spectrum of WISE 1741-4642 shows a distinctly peaked $H$-band continuum, a well known characteristic of young brown dwarfs (Lucas et al. 2001; Figures 1-3). The shape of the $H$-band continuum is determined by differing sources of opacity production that depend on an object's surface gravity (Rice et al. 2011; Barman et al. 2011; Marley et al. 2012). For higher gravities, the $H$-band shape is dominated by collision-induced absorption (CIA) opacity or cloud opacity, which results in a rounded appearance. For a low surface gravity object, cloud opacity and CIA become less significant, and the $H$-band shape is governed by molecular bands around 


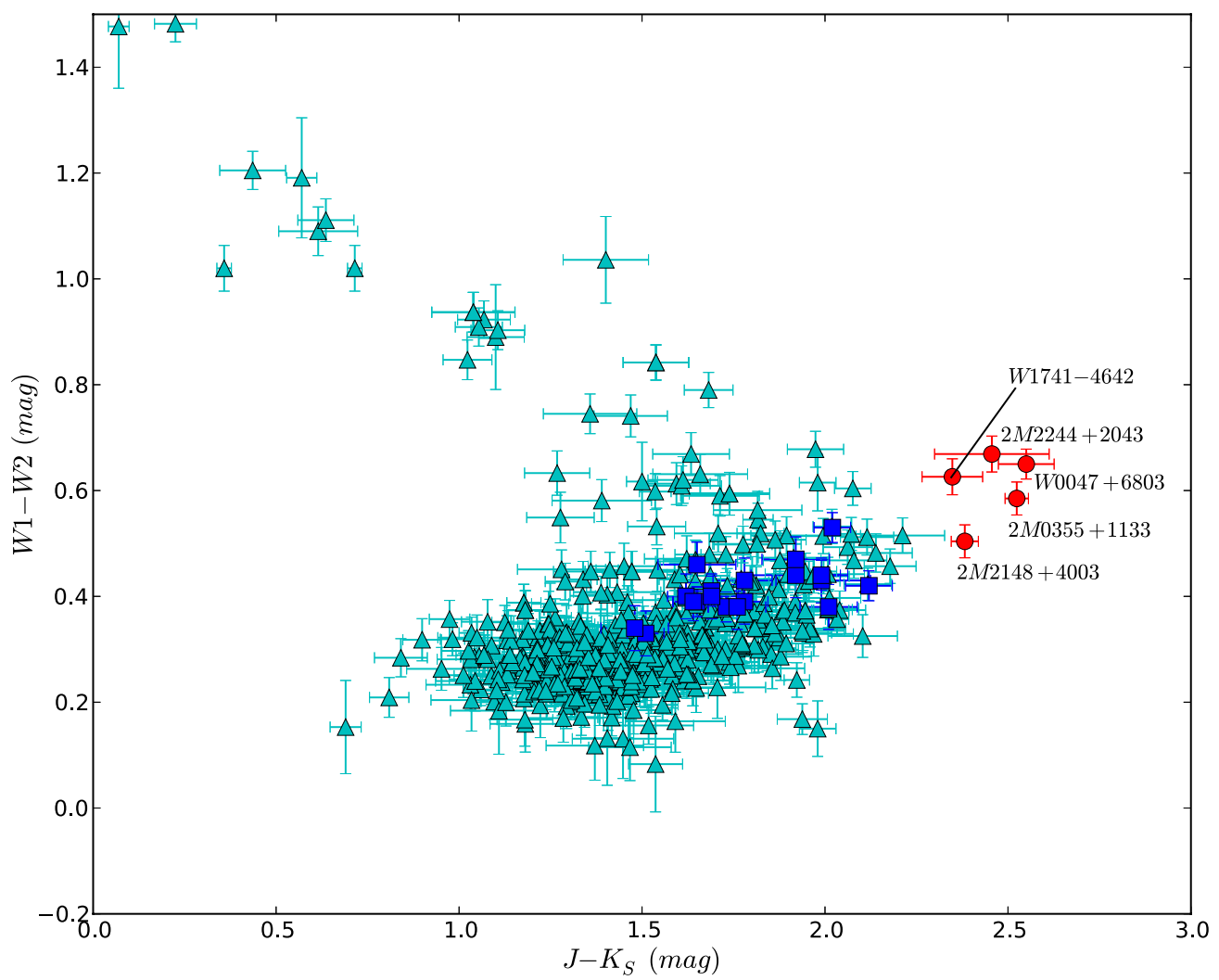

Figure 5. 2MASS $J-K_{S}$ vs. WISE $W 1-W 2$ colors for dwarfs with photometric uncertainties $\leqslant 0.1 \mathrm{mag}$ from http://www.dwarfarchives.org (cyan triangles). WISE 1741-4642 and red L dwarfs 2M2148+4003, 2M2244+2043, 2M0355+1133, and W0047+6803 are shown as red circles. Blue squares represent L $\gamma$ dwarfs from Table 3 of Faherty et al. (2013), the majority of which have optical spectral types earlier than L5.

(A color version of this figure is available in the online journal.)

$1.7 \mu \mathrm{m}$, most notably $\mathrm{H}_{2} \mathrm{O}$, which results in a peaked triangular appearance.

WISE 1741-4642 also shows a redder $J-K_{S}$ color $\left(J-K_{S}=2.35 \pm 0.08 \mathrm{mag}\right)$ and $W 1-W 2$ color $(W 1-W 2=$ $0.63 \pm 0.03 \mathrm{mag}$ ) compared to objects of similar spectral type (Figures 2 and 5). Faherty et al. (2013) demonstrate that the $J-K_{S}$ and $W 1-W 2$ colors of low surface gravity dwarfs deviate from the mean population. The triangular $H$-band shape and red $J-K_{S}$ and $W 1-W 2$ colors of WISE 1741-4642 are features typical of young dwarfs and are often caused by low surface gravity. Kirkpatrick et al. (2008) show that gravity-sensitive spectral features are only evident in objects with Pleiades ages or younger. Therefore, the ability to distinguish low-gravity spectral features implies an age $<100 \mathrm{Myr}$. Following the example of Cruz et al. (2009), we take $10 \mathrm{Myr}$ as a lower age limit because WISE $1741-4642$ is isolated from young, dense, star-forming regions. This age is also an approximate lower limit for the ages of young nearby moving groups.

Allers \& Liu (2013) define several gravity sensitive spectral indices that take advantage of the gravity sensitivity of $\mathrm{FeH}$ bands (centered on the 0.99 and $1.20 \mu \mathrm{m}$ bandheads), VO absorption (centered on the $1.06 \mu \mathrm{m}$ bandhead), the $\mathrm{K}_{\mathrm{I}}$ line at $1.14 \mu \mathrm{m}$, and the $H$-band continuum shape. We note here that the alkali and molecular band indices are most useful for spectral types between $\sim$ M9 and L5, and are degenerate for our predicted spectral type of L7. We can, however, determine the $H$-cont index, which is designed to quantify the $H$-band continuum shape. We calculated an $H$-cont value of 0.955 for our SpeX spectrum, which follows the low-gravity sequence, well separated from the location of normal field dwarfs (see
Figure 24 of Allers \& Liu 2013). For the similarly red L7.5 (pec) dwarf W0047+6803, we calculate an $H$-cont value of 0.963 . We can also utilize the K I pseudo-equivalent width (EW) definitions of Allers \& Liu (2013) using our moderate-resolution FIRE spectrum. Alkali lines are known to be gravity sensitive, and can appear narrower than those seen in normal field dwarfs because of the lack of pressure broadened wings (Kirkpatrick et al. 2006). We first smoothed our FIRE spectrum with a Gaussian kernel to a resolution $(R \sim 1500)$ similar to those inspected in Allers \& Liu (2013). We measured pseudo-EW values for the 1.169, 1.177, and $1.252 \mu \mathrm{m} \mathrm{K} \mathrm{I}$ lines of $0.93,1.89$, and $0.86 \AA$, respectively. We similarly measure the pseudo-EWs of W0047+6803 (3.31, 2.62, and $1.16 \AA$ ) and W0355+1133 (2.58, 2.59, and $0.98 \AA)$. All three measured pseudo-EWs are consistent with a low-gravity object (see Figure 23 and Table 10 of Allers \& Liu 2013). Figures 6 and 7 display the K I spectral line regions of WISE 1741-4642 compared with the red L dwarfs W0047+6803 and W0355+1133. The FIRE spectrum has been smoothed in both figures to a resolution comparable to each comparison spectrum. Figures 6 and 7 show that WISE 1741-4642 has KI line strengths comparable to both W0047+6803 and W0355+1133. We also compared the $\mathrm{K}_{\mathrm{I}}$ lines with field brown dwarfs from the NIRSPEC Brown Dwarf Spectroscopic Survey (BDSS; McLean et al. 2007). Comparisons with the L5 field dwarf 2M1507-16 and the L7 field dwarf DENIS 0205-11 are shown in Figures 8 and 9. Each figure shows that the K I lines of WISE 1741-4642 are weaker and/or narrower than those lines seen in field dwarfs of a similar spectral type. We note that $\mathrm{K}_{\mathrm{I}}$ line strengths are also sensitive to temperature differences, which may contribute to the dissimilarity of the spectra. 

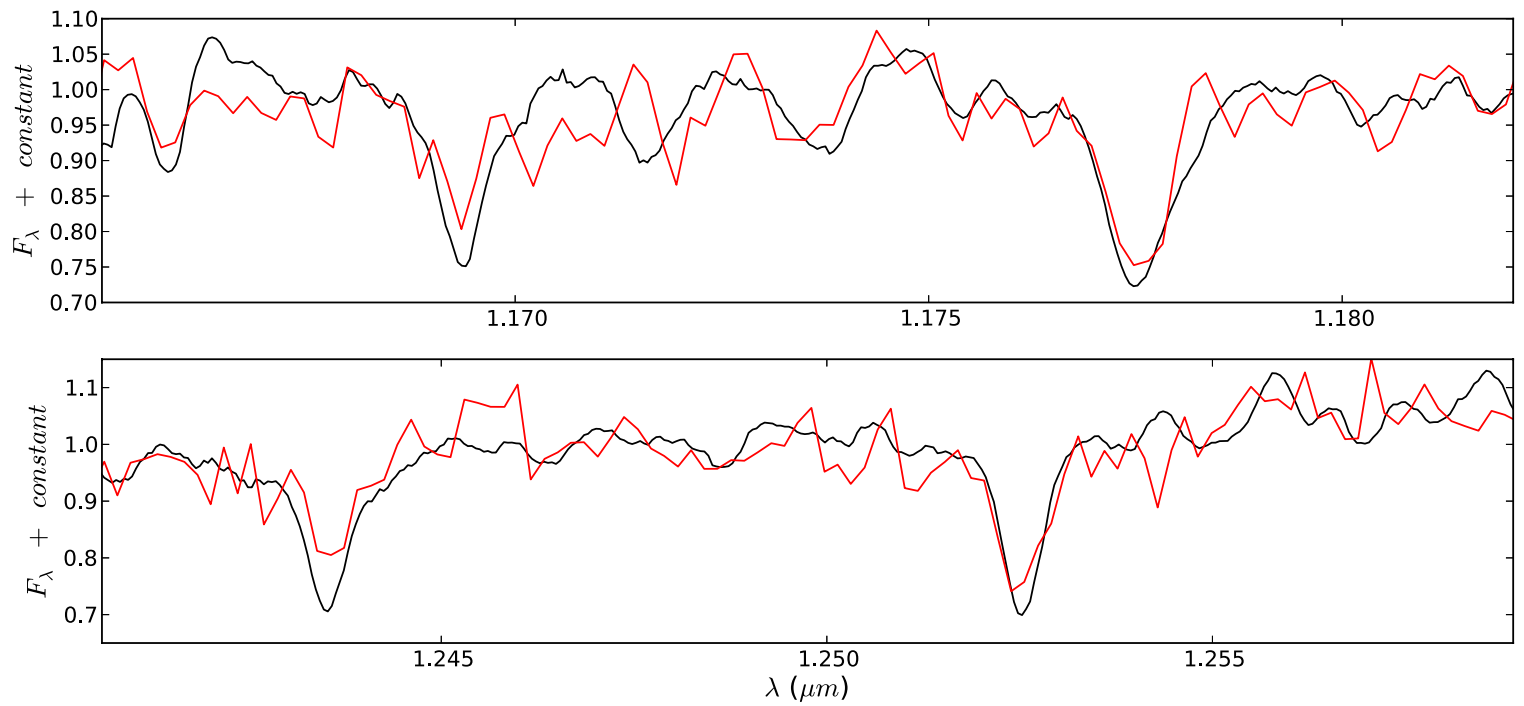

Figure 6. K I line comparison of the WISE 1741-4642 FIRE spectrum (black) with the red L7.5 dwarf W0047+6803 (red; Gizis et al. 2012). The FIRE spectrum was smoothed with a Gaussian kernel to the resolution of the comparison spectrum. The flux of both spectra were normalized to the continuum.

(A color version of this figure is available in the online journal.)
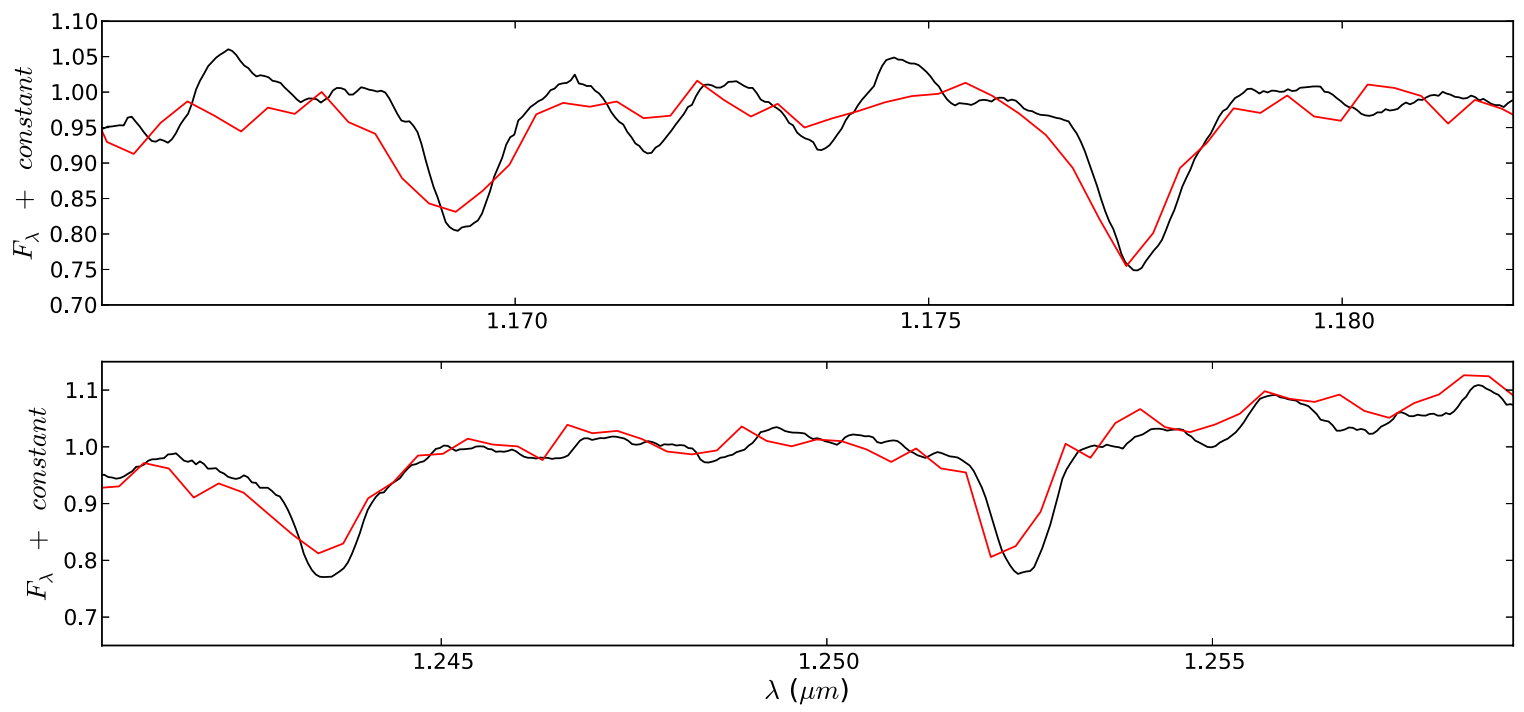

Figure 7. K I line comparison of the WISE 1741-4642 FIRE spectrum (black) with the L5 $\gamma$ dwarf 2M0355+1133 (red; Faherty et al. 2013). The FIRE spectrum was smoothed with a Gaussian kernel to the resolution of the comparison spectrum. The flux of both spectra were normalized to the continuum.

(A color version of this figure is available in the online journal.)

Canty et al. (2013) show that the $K$-band continuum shape can easily distinguish young ( $\leqslant 10 \mathrm{Myr}$ ) brown dwarfs from an older field population, and can be used with confidence even with low resolution spectra. We note that the sample used in Canty et al. (2013) has a dearth of objects with intermediate ages ( $>10 \mathrm{Myr}$ and less than the age of field dwarfs) and the effectiveness of the $\mathrm{H}_{2}(K)$ index in this age range is not well constrained. They define an $\mathrm{H}_{2}(K)$ index to quantify the $K$-band continuum slope, given as

$$
\mathrm{H}_{2}(K)=\frac{F_{\lambda}(2.17 \mu \mathrm{m})}{F_{\lambda}(2.24 \mu \mathrm{m})},
$$

where $F_{\lambda}(2.17 \mu \mathrm{m})$ and $F_{\lambda}(2.24 \mu \mathrm{m})$ are the median fluxes over a total range of $0.02 \mu \mathrm{m}$ centered at 2.17 and $2.24 \mu \mathrm{m}$, respectively. We calculated the $\mathrm{H}_{2}(K)$ index of WISE 1741-4642 and found a value of 1.029. However, we note that the analysis of the $K$-band continuum shape by Canty et al. (2013) for young brown dwarfs was performed for spectral types $\leqslant$ L0. To extend the work of Canty et al. (2013) into the L dwarf regime, we gathered all available spectra from the Spex Prism Spectral Library for which a resolving power $\geqslant 120$ is available. Since many of the given or published spectral types were not determined homogeneously, we independently derived near-infrared spectral types for each available spectrum based on the Kirkpatrick et al. (2010) L dwarf classification scheme (see the Appendix). The $\mathrm{H}_{2}(K)$ index of each $\mathrm{L}$ dwarf was calculated, and is shown in Figure 10 and given in Table 2. Any spectrum found to be especially noisy was not included in this analysis. While a spectral type dependence is seen past L0 (the $\mathrm{H}_{2}(K)$ index generally increases with increasing spectral type), WISE 1741-44642 and the red L dwarfs $2 \mathrm{M} 0355+1133$ and $2 \mathrm{M} 0047+6803$ are seen to deviate from the field population. Also showing strong deviations below their near-infrared spectral types are the young, red L6 dwarf 2MASS J214816.28+400359.3 (Looper et al. 2008b), the young, red L2 dwarf 2MASS J014158.23-463357.4 (Kirkpatrick et al. 

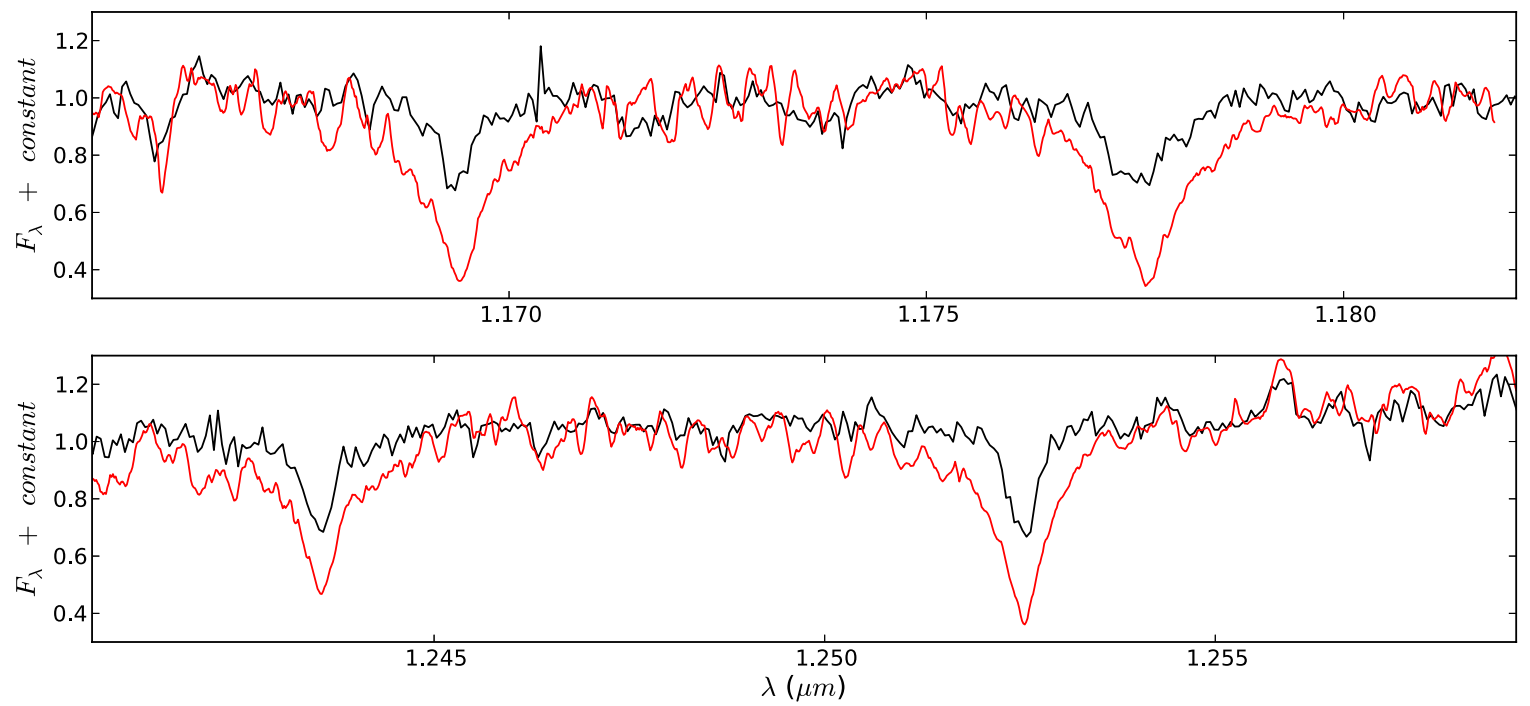

Figure 8. K I line comparison of the WISE 1741-4642 FIRE spectrum (black) with the L5 dwarf 2M1507-16 spectrum from the BDSS (red; McLean et al. 2007). The NIRSPEC spectrum was smoothed with a Gaussian kernel to the resolution of the FIRE spectrum. The flux of both spectra were normalized to the continuum. (A color version of this figure is available in the online journal.)
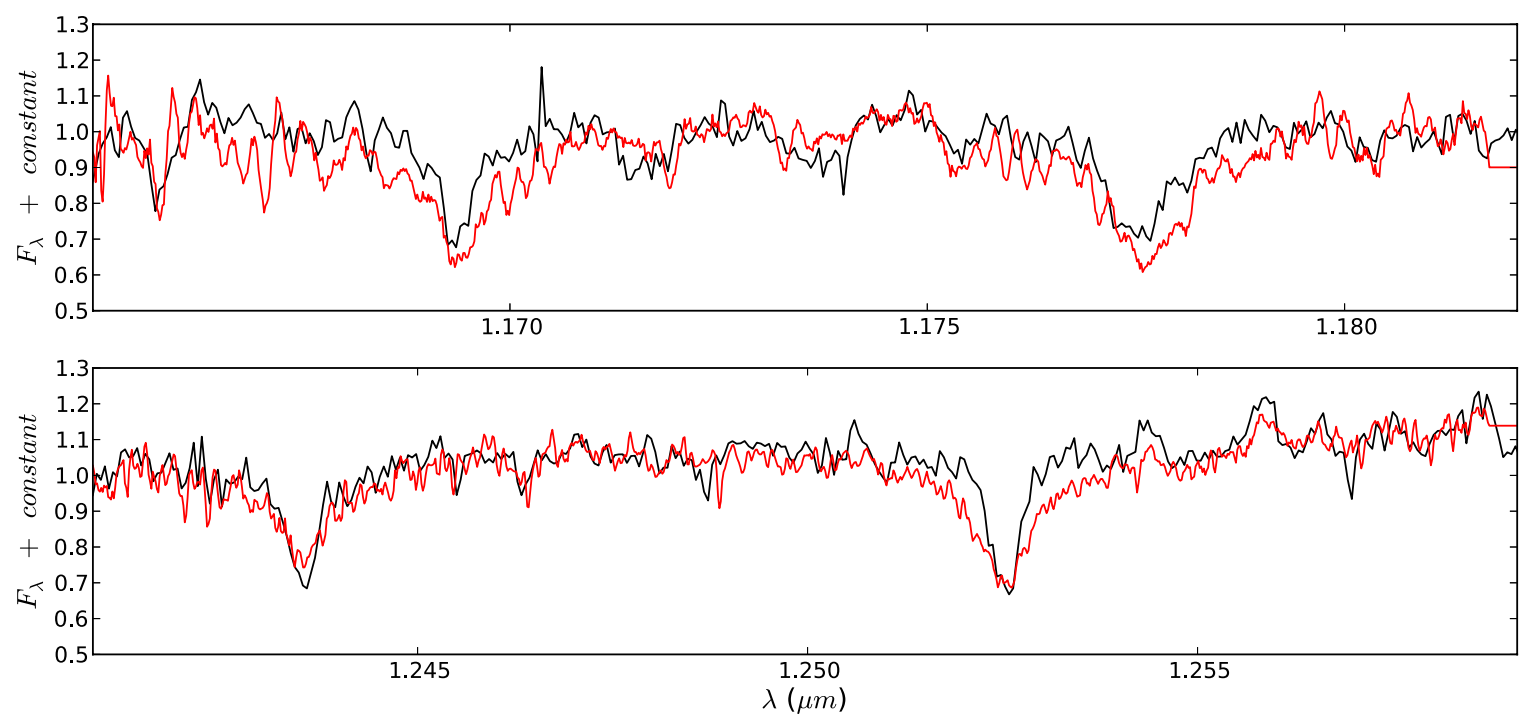

Figure 9. K I line comparison of the WISE 1741-4642 FIRE spectrum (black) with the L7 dwarf DENIS0205-11 spectrum from the BDSS (red; McLean et al. 2007). The NIRSPEC spectrum was smoothed with a Gaussian kernel to the resolution of the FIRE spectrum. The flux of both spectra were normalized to the continuum.

(A color version of this figure is available in the online journal.)

2006), the low-gravity L dwarf 2MASS J224953.45+004404.6 $\left(\mathrm{SpT}_{\text {opt }}=\mathrm{L} 4 \gamma\right.$; Faherty et al. 2013), and the red L8 dwarf SDSSp J010752.33+004156.1 (Geballe et al. 2002).

In summary, WISE 1741-4642 shows several traits, such as a peaked $H$-band continuum shape, red near-infrared colors, and a low $\mathrm{H}_{2}(K)$ index, that are characteristic of a young age.

\subsubsection{Model Age and Mass}

We compared the position of WISE 1741-4642 in $T_{\text {eff }}$ versus $\log g$ space with the "COND" (Baraffe et al. 2003), "DUSTY" (Chabrier et al. 2000), and Saumon \& Marley (2008) cloudy and cloudless low-mass evolutionary models (discussed in Section 3.4.1). We note here that the spectral models of Allard et al. (2011) and the low-mass evolutionary models used in Section 3.4 are not self-consistent. However, such a comparison is the only available procedure for determining the physical characteristics of WISE 1741-4642. Figures 11 and 12 show that the best fitting model spectrum $\left(T_{\text {eff }}=1600\right.$, log $\left.g=3.5\right)$ suggests an age $\leqslant 5 \mathrm{Myr}$, but there are no regions of ongoing star formation within $30 \mathrm{pc}$. Because this is unphysical for WISE 1741-4642, we use the next two best fitting models. For $T_{\text {eff }}=1450 \pm 100 \mathrm{~K}$ and $\log g=4.0 \pm 0.25$, the "COND" and "DUSTY" models suggest an age between 5 and $100 \mathrm{Myr}$ (Figure 11). The Saumon \& Marley (2008) models suggest an age of 3-100 Myr (Figure 12). Both model ages are consistent with the age implied by the near-infrared spectral characteristics of WISE 1741-4642, and we take 10-100 Myr as our final age estimate for WISE 1741-4642. We take $10 \mathrm{Myr}$ as a lower age limit because WISE $1741-4642$ is isolated from young, dense, star-forming regions. Even though the best fitting model was deemed unphysical, this type of object has the potential to anchor low-mass evolutionary models for young ages.

The same models and spectral model parameters are used to estimate the mass of WISE 1741-4642. The "COND" and "DUSTY" models suggest a mass range of 5-21 $M_{\text {Jup }}$, while the Saumon \& Marley (2008) models suggest a mass range of 


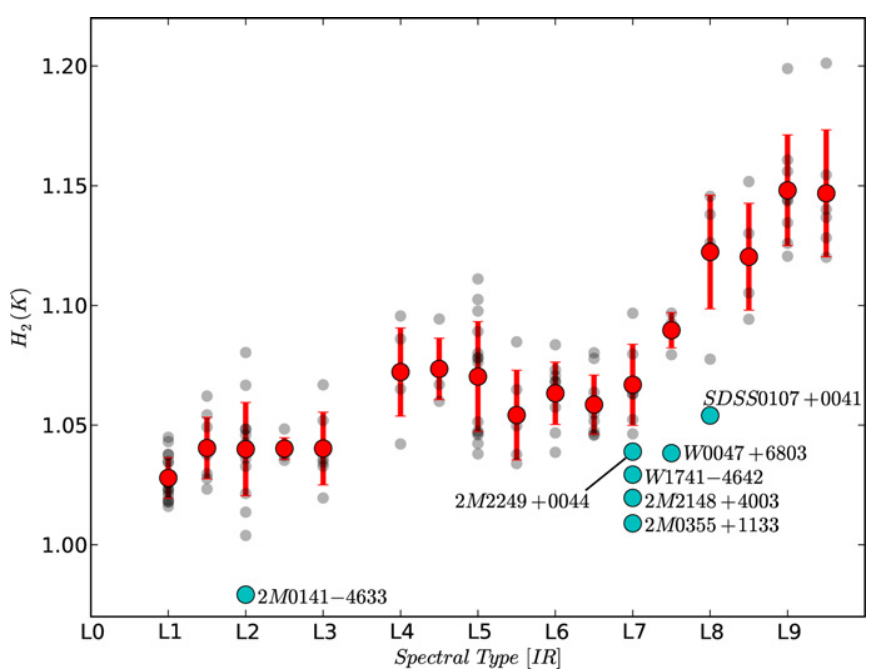

Figure 10. $\mathrm{H}_{2}(K)$ index as a function of spectral type for $\mathrm{L}$ dwarfs from the SpeX Prism Spectral Library based on our new spectral typing system. Red points indicate the mean $\mathrm{H}_{2}(K)$ index and standard deviation for $\mathrm{L}$ dwarfs binned by 0.5 subtype. The young and/or red L dwarfs $2 \mathrm{M} 2148+4003,2 \mathrm{M} 0355+1133$, 2M0141-4633, 2M2249+0044, W0047-6803, and SDSS 0107+0041, as well as WISE 1741-4642, are individually labeled.

(A color version of this figure is available in the online journal.)

4-15 $M_{\text {Jup }}$ (Figures 11 and 12 ). We take $4-21 M_{\text {Jup }}$ as our final mass estimate.

\subsection{Kinematic Analysis of WISE 1741-4642}

Because WISE 1741-4642 is located in the southern hemisphere, where many young moving groups and associations reside, we considered the possibility that it belongs to one of these. Although a full moving group membership analysis requires accurate parallax, proper motion, and radial velocity measurements, we may still explore how probable nearby moving group membership is for WISE $1741-4642$. We calculate proper motions in right ascension and declination using the WISE All-Sky and 2MASS positions of WISE 1741-4642 (listed in Table 1). Using a distance estimate of $10-30 \mathrm{pc}$ and the proper motions given in Table 1 , we calculated $U V W^{8}$ space motions for WISE 1741-4642 for a range of radial velocities. Then we compared the resulting $U V W$ space motions to the "good box" from Zuckerman \& Song (2004), a region of $U V W$ space where nearby moving group members reside. Because $U V W$ space motions depend on an accurate distance measurement, we calculated them using 10, 20, and $30 \mathrm{pc}$ as the distance to WISE 1741-4642. There is a range of acceptable radial velocities that would put WISE 1741-4642 in the $U V W$ space that coincides with nearby young moving groups, which is illustrated in Figure 13. Figure 13 also shows that WISE $1741-4642$ is much more likely to belong to a nearby young moving group if its distance is 10 or $20 \mathrm{pc}$, rather than $30 \mathrm{pc}$. As shown in Section 3.5.1, the largest photometric distance estimates come from the relations of Dupuy \& Liu (2012) and Looper et al. (2008a) and the $J$-band magnitude of WISE 1741-4642, which may be underluminous.

In addition, we also took advantage of the convergent point analysis and the Bayesian young nearby association membership probability tools developed by Rodriguez et al. (2013) and

\footnotetext{
$8 U V W$ space motions are calculated using the measured radial velocity. $U V W$ are defined with respect to the Sun. $U$ is positive toward the Galactic center, $V$ is positive in the direction of Galactic rotation, and $W$ is positive toward the north Galactic pole.
}
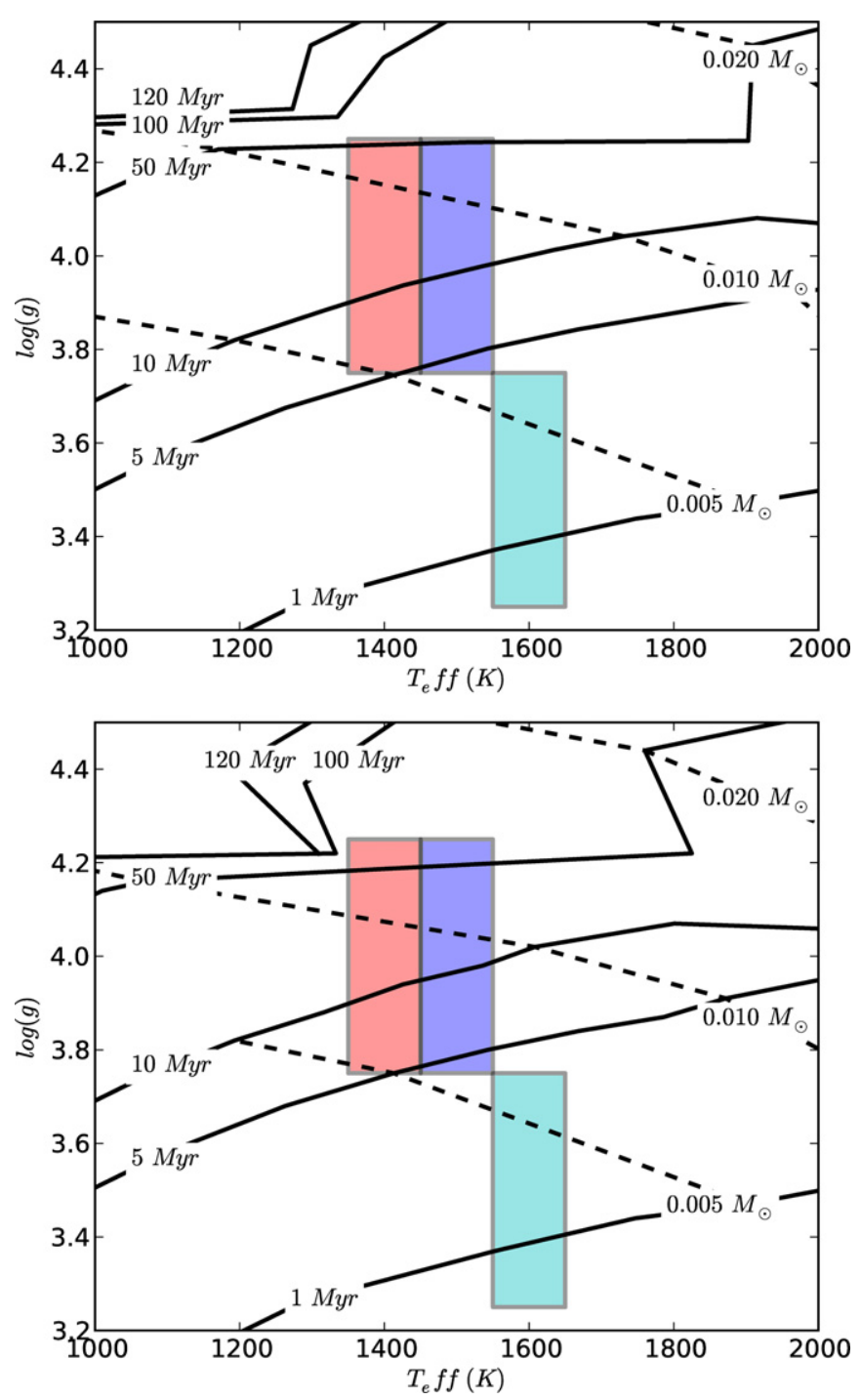

Figure 11. Top: theoretical "COND" $\log g$ vs. $T_{\text {eff }}$ evolutionary tracks from Baraffe et al. (2003). Isochrones of 1, 5, 10, 50, 100, and $120 \mathrm{Myr}$ are shown by solid lines from right to left. Curves of constant mass from $0.005,0.010$, and $0.020 M_{\odot}\left(5,10\right.$, and $\left.21 M_{\mathrm{Jup}}\right)$ are shown by dashed lines from left to right. The red, blue, and cyan shaded regions represent the three best model spectrum fits: $T_{\text {eff }}=1400 \mathrm{~K}$ and $\log g=4.0$ (red), $T_{\text {eff }}=1500 \mathrm{~K}$ and $\log g=4.0$ (blue), and $T_{\text {eff }}=1600 \mathrm{~K}$ and $\log g=3.5$ (cyan). The colors match those in Figure 6. Bottom: same as on the left for the theoretical "DUSTY" $\log g$ vs. $T_{\text {eff }}$ evolutionary tracks from Chabrier et al. (2000).

(A color version of this figure is available in the online journal.)

Malo et al. (2013), respectively. As both authors state clearly, high probabilities do not guarantee young association membership. However, these tools can aid in illustrating the likelihood of nearby moving group membership for WISE 1741-4642.

The convergent point analysis tool of Rodriguez et al. (2013) uses the position and proper motion of an astronomical body to calculate parallel and perpendicular proper motion components relative to the convergence point of several nearby young associations. WISE 1741-4642 membership probabilities for the TW Hydrae Association (TWA), the Tucana-Horologium association (Tuc-Hor), the Carina-Near moving group, and the Columba moving group are all less than $10 \%$. Membership probabilities for the $\beta$ Pictoris and AB Doradus associations are 94.2 and $91.9 \%$, respectively. Furthermore, the convergent point analysis tool returns the ideal kinematic distances and radial velocities for membership in each group. The kinematic distance 

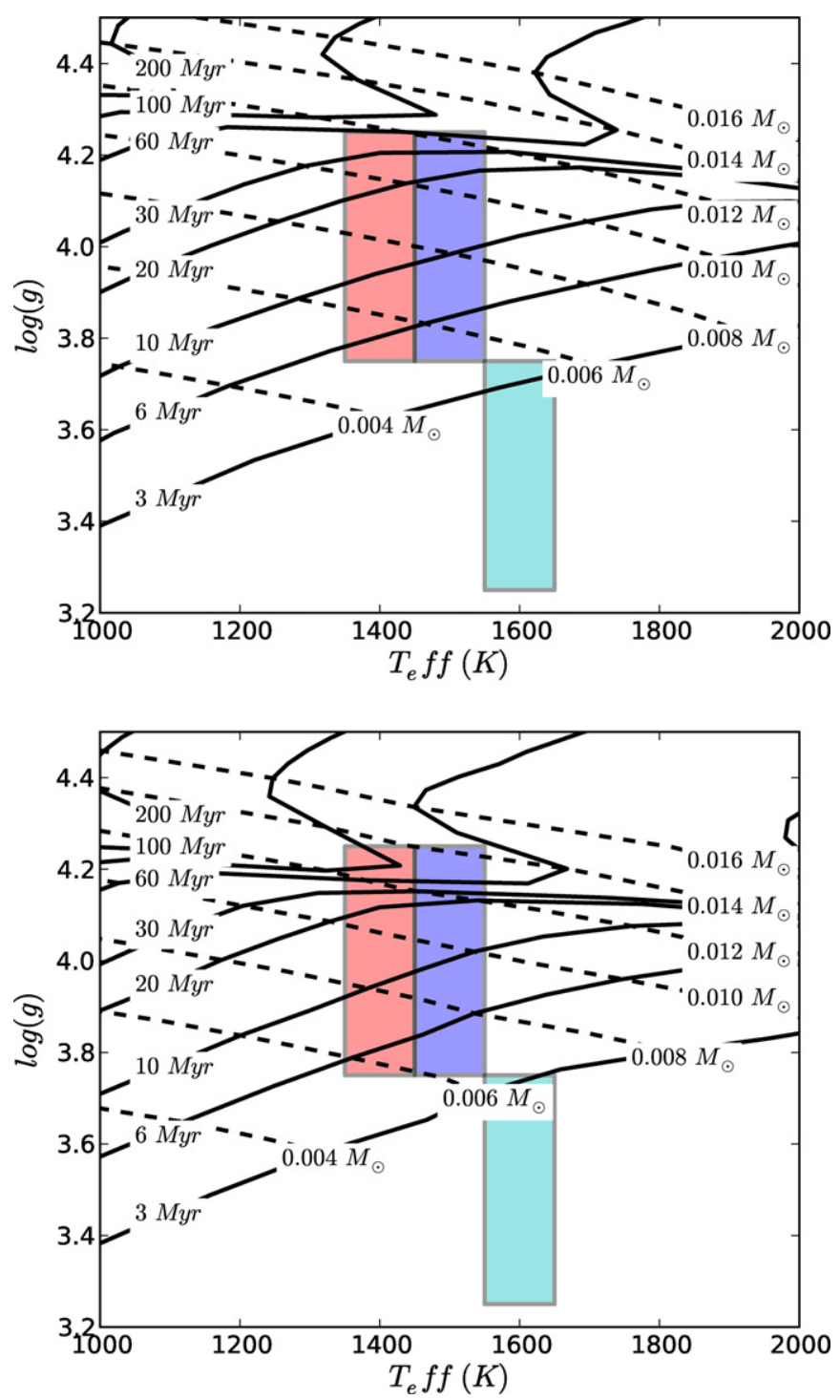

Figure 12. Top: theoretical (cloudless) $\log g$ vs. $T_{\text {eff }}$ evolutionary tracks from Saumon \& Marley (2008). Isochrones of 3, 6, 10, 20, 30, 60, 100, and $200 \mathrm{Myr}$ are shown by solid lines from right to left. Curves of constant mass from $0.004-0.016 M_{\odot}\left(4-17 M_{\text {Jup }}\right)$ in steps of $0.002 M_{\odot}$ are shown by dashed lines from left to right. The red, blue, and cyan shaded regions represent the three best model spectrum fits: $T_{\text {eff }}=1400 \mathrm{~K}$ and $\log g=4.0$ (red), $T_{\text {eff }}=1500 \mathrm{~K}$ and $\log g=4.0$ (blue), and $T_{\text {eff }}=1600 \mathrm{~K}$ and $\log g=3.5$ (cyan). The colors match those in Figure 6. Bottom: same as on the top for the theoretical (cloudy; $\left.f_{\text {sed }}=2\right) \log g$ vs. $T_{\text {eff }}$ evolutionary tracks from Saumon \& Marley (2008).

(A color version of this figure is available in the online journal.)

is predicted to be $12.3 \mathrm{pc}$ for $\beta$ Pictoris and $19.1 \mathrm{pc}$ for AB Doradus, distances that match well with our photometric distance estimates. The predicted radial velocities are $-5.4 \mathrm{~km} \mathrm{~s}^{-1}$ and $2.1 \mathrm{~km} \mathrm{~s}^{-1}$ for $\beta$ Pictoris and AB Doradus, respectively.

The Bayesian Analysis for Nearby Young AssociatioNs tool (BANYAN; Malo et al. 2013) uses Bayesian inference to determine association membership probabilities, predicted radial velocities, and the most probable distances for several young nearby associations. Unlike the convergent point analysis tool of Rodriguez et al. (2013), the BANYAN tool determines membership probabilities so that they sum to $100 \%$. Using the WISE All-sky catalog position and the proper motions provided in Table 1, the BANYAN tool determined that WISE 1741-4642 has a $0 \%$ probability of belonging to TWA, Tuc-Hor, Columba, and Carina. Membership probabilities for $\beta$ Pictoris, AB Do-
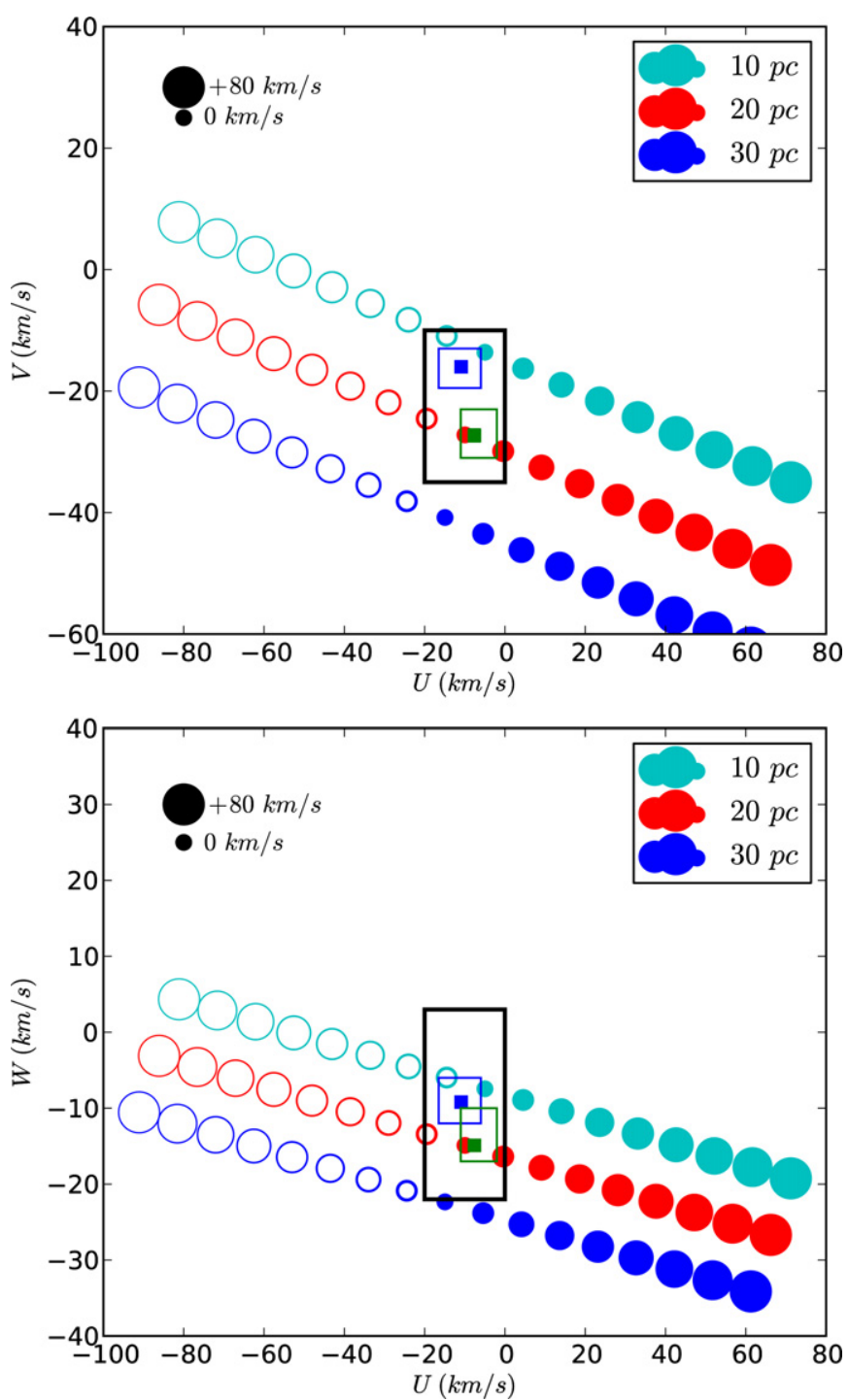

Figure 13. $U V W$ space velocities for WISE 1741-4642 for a range of radial velocities ( -80 to $+80 \mathrm{~km} \mathrm{~s}^{-1}$ in $10 \mathrm{~km} \mathrm{~s}^{-1}$ steps) for distances of 10,20 , and $30 \mathrm{pc}$. Negative radial velocity values are represented by open circles. The black square indicates the "good box" from Zuckerman \& Song (2004). The blue and green empty boxes indicate the extent of highly probably members from Torres et al. (2008) for $\beta$ Pictoris and the AB Dor association, respectively. Small blue and green filled squares represent the core $U V W$ values of $\beta$ Pictoris and $\mathrm{AB}$ Doradus as defined in Faherty et al. (2013).

(A color version of this figure is available in the online journal.)

radus, Argus, and old field stars are $40.75 \%, 43.45 \%, 15.34 \%$, and $0.47 \%$, respectively (Argus is not considered by the convergent point analysis tool of Rodriguez et al. 2013). As with the convergent point analysis tool, the BANYAN tool claims a high likelihood that WISE 1741-4642 belongs to either $\beta$ Pictoris or AB Doradus ( $\sim 84 \%$ total). Statistical distances are in excellent agreement with those from the convergent point method as well; $13.0 \mathrm{pc}$ for $\beta$ Pictoris and $19.5 \mathrm{pc}$ for AB Doradus, again consistent with our photometric distance estimates. The predicted radial velocities from BANYAN are $-4.7 \mathrm{~km} \mathrm{~s}^{-1}$ and $+2.57 \mathrm{~km} \mathrm{~s}^{-1}$ for $\beta$ Pictoris and AB Doradus, respectively.

We estimated the radial velocity of WISE 1741-4642 from our FIRE spectrum using measured $\mathrm{K}_{\mathrm{I}}$ central wavelengths compared to the rest wavelengths found in Rayner et al. (2009). We measured the central wavelength for each of the four $\mathrm{K}$ I lines at $1.16934,1.17761,1.24356$, and $1.252566 \mu \mathrm{m}$ and from these calculated a radial velocity, corrected for the heliocentric radial 

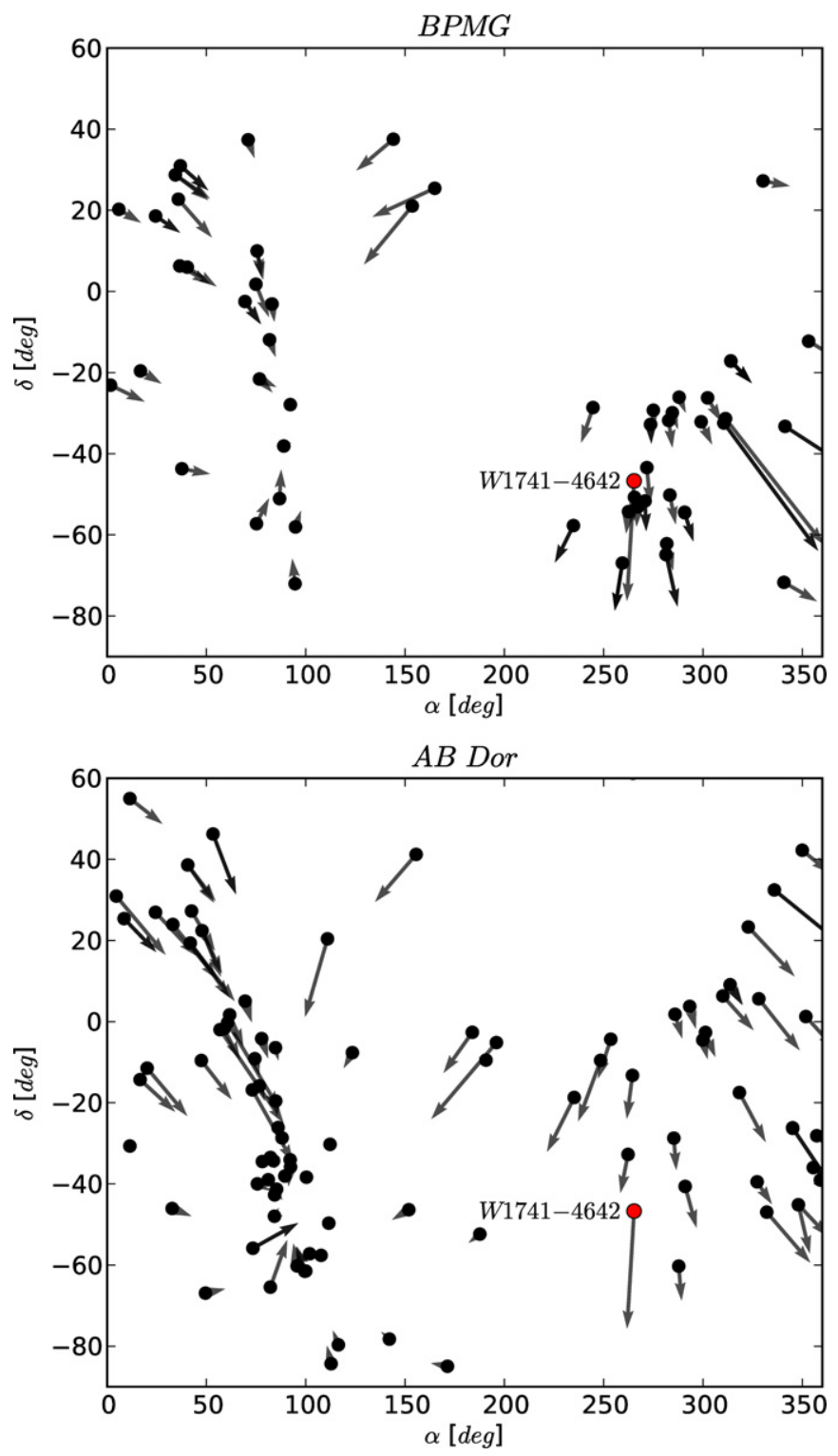

Figure 14. Left: the positions and proper motion of WISE 1741-4642 along with members of the Beta Pictoris moving group. Right: the positions and proper motion of WISE 1741-4642 along with members of the AB Doradus moving group.

(A color version of this figure is available in the online journal.)

velocity, of $-5.7 \pm 5.1 \mathrm{~km} \mathrm{~s}^{-1}$. While uncertainties using this method are large, a radial velocity significantly discrepant from those predicted for WISE $1741-4642$ if it is a $\beta$ Pictoris or AB Doradus member could have ruled out potential membership. Our measured radial velocity range does not rule out $\beta$ Pictoris or AB Doradus membership. We also examined the XYZ position of WISE 1741-4642 using the WISE All-Sky position and our distance estimate of $10-30 \mathrm{pc}$. We find XYZ values of $(9.5,-2.7,-1.5),(19.0,-5.3,-2.9)$, and $(28.6,-8.0,-4.4) \mathrm{pc}$ for distances of 10,20 , and $30 \mathrm{pc}$, respectively. Comparing these values to the mean $\mathrm{XYZ}$ positions and dispersions for $\beta$ Pictoris $(9.27 \pm 31.71,-5.96 \pm 15.19,-13.59 \pm 8.22) \mathrm{pc}$ and the $\mathrm{AB}$ Doradus association $(-2.37 \pm 20.03,1.48 \pm 18.83,-15.62 \pm$ 16.59) pc from Malo et al. (2013), we find that the XYZ positions of WISE 1741-4642 are consistent with known members of the $\beta$ Pictoris and $\mathrm{AB}$ Doradus associations.

Given the youthful characteristics of the near-infrared spectrum of WISE 1741-4642 and the good agreement of its space motion with the $\beta$ Pictoris and AB Doradus associations, we believe there is a strong possibility of moving group membership. The position and proper motion of WISE 1741-4642 along with known members of $\beta$ Pictoris and AB Dordados members from Schneider (2013) is displayed in Figure 14. Accurate parallax and radial velocity measurements will aid in clearing up any young association membership ambiguity.

\section{CONCLUSIONS}

We have shown through an analysis of the near-infrared spectrum and photometric colors of WISE 1741-4642 that it shows characteristics indicative of an age of 10-100 Myr. Since WISE $1741-4642$ is young, it is less massive than the majority of objects with similar spectral types, which typically have ages greater than $1 \mathrm{Gyr}$ (assuming spectral type correlates with effective temperature). We estimated a spectral type of $\mathrm{L} 7 \pm 2$ by a comparison of the near-infrared spectrum to spectral standards and other red L dwarfs. We determined an effective temperature of $1450 \pm 100 \mathrm{~K}$ and $\log g$ of $4.0 \pm 0.25$ from a comparison to spectral models. From the $T_{\text {eff }}$ and $\log g$ estimates, we estimate the mass of WISE 1741-4642 to be 4-21 $M_{\text {Jup }}$. We also explored the possibility that WISE 1741-4642 is a member of one of the established nearby, young moving groups or associations. We found that membership of WISE 1741-4642 in the $\beta$ Pictoris or AB Doradus moving groups is a possibility deserving follow-up observations. Future parallax and radial velocity measurements can confirm any association membership.

We would like to acknowledge the recently discovered young late-type L dwarf (and likely $\beta$ Pictoris Moving Group member) PSO J318.5338-228603 (Liu et al. 2013), which was published during the refereeing phase of this manuscript. Its extremely red near-infrared colors and low estimated mass make it another great young, low-mass object for substellar evolution studies and exoplanetary comparisons.

This research has made use of the SIMBAD database and VizieR catalog access tool, operated at CDS, Strasbourg, France. This publication makes use of data products from the Two Micron All Sky Survey, which is a joint project of the University of Massachusetts and the Infrared Processing and Analysis Center/California Institute of Technology, funded by the National Aeronautics and Space Administration and the National Science Foundation, and the Wide-field Infrared Survey Explorer, which is a joint project of the University of California, Los Angeles, and the Jet Propulsion Laboratory/California Institute of Technology, funded by the National Aeronautics and Space Administration. This research has made use of the NASA/IPAC Infrared Science Archive, which is operated by the Jet Propulsion Laboratory, California Institute of Technology, under contract with the National Aeronautics and Space Administration. This research has benefitted from the SpeX Prism Spectral Libraries, maintained by Adam Burgasser at http://pono.ucsd.edu/ adam/browndwarfs/spexprism. This research has benefitted from the $\mathrm{M}, \mathrm{L}, \mathrm{T}$, and $\mathrm{Y}$ dwarf compendium housed at dwarfarchives.org. G.N.M. was a Visiting Astronomer at the Infrared Telescope Facility, which is operated by the University of Hawaii under Cooperative Agreement No. NNX-08AE38A with the National Aeronautics and Space Administration, Science Mission Directorate, Planetary Astronomy Program. The Brown Dwarf Spectroscopic Survey is hosted by UCLA and provided an essential comparison library for our moderate resolution spectroscopy. 
Table 2

SpeX Prism Spectral Library Near-infrared L Dwarf Spectral Types

\begin{tabular}{|c|c|c|c|c|c|c|}
\hline 2MASS Name & $\begin{array}{l}\mathrm{SpT}_{\mathrm{opt}} \\
\text { (Pub.) }\end{array}$ & $\begin{array}{l}\mathrm{SpT}_{\mathrm{NIR}} \\
\text { (Pub.) }\end{array}$ & $\begin{array}{c}\mathrm{SpT}_{\mathrm{IR}} \\
\text { (Adopted) }\end{array}$ & $\begin{array}{l}\mathrm{H}_{2} \\
(\mathrm{~K})\end{array}$ & Ref. & Note \\
\hline 2M0004-4044 & L5 & L4.5 & L5 & 1.051 & 1 & \\
\hline $2 \mathrm{M} 0015+2959$ & L7 & L7.5 (pec) & L8.5 & $\ldots$ & 2 & \\
\hline $2 \mathrm{M} 0016-4056$ & L3.5 & $\ldots$ & L5.5 & 1.034 & 3 & \\
\hline $2 \mathrm{M} 0030-1450$ & L7 & $\ldots$ & L6.5 & 1.052 & 3 & \\
\hline $2 \mathrm{M} 0032+1410$ & $\ldots$ & L8 & L9 & 1.199 & 3 & \\
\hline $2 \mathrm{M} 0036+1821$ & L3.5 & L4 & L1.5 & 1.062 & 4 & \\
\hline $2 \mathrm{M} 0036+2413$ & $\ldots$ & L5.5 & L6 & $\ldots$ & 5 & \\
\hline $2 \mathrm{M} 0051-1544$ & L3.5 & $\ldots$ & L6 & 1.047 & 3 & \\
\hline $2 \mathrm{M} 0053-3631$ & L3.5 & $\ldots$ & L4 & 1.072 & 3 & \\
\hline $2 \mathrm{M} 0103+1935$ & L6 & L7 & L7 & 1.063 & 6 & \\
\hline $2 \mathrm{M} 0107+0041$ & L8 & L5.5 & L8 & $\ldots$ & 3 & Red \\
\hline 2M0130-4445 & $\ldots$ & L6 & L7.5 & $\ldots$ & 7 & \\
\hline $2 \mathrm{M} 0134+0508$ & $\ldots$ & L1 & $\mathrm{L} 1$ & 1.022 & 2 & \\
\hline $2 \mathrm{M} 0141-1601$ & $\ldots$ & L7: & L5.5 & $\ldots$ & 2 & \\
\hline $2 \mathrm{M} 0141-4633$ & $\mathrm{~L} 0 \gamma$ & L0 (pec) & $\mathrm{L} 2$ & $\ldots$ & 22 & Red \\
\hline 2M0144-0716 & L5 & $\ldots$ & L6.5 & 1.046 & 4 & \\
\hline $2 \mathrm{M} 0147+4731$ & $\ldots$ & L1.5 & $\mathrm{L} 1$ & 1.024 & 2 & \\
\hline $2 \mathrm{M} 0205+1251$ & L5 & $\ldots$ & L6.5 & 1.046 & 8 & \\
\hline 2M0205-0795 & $\ldots$ & $\mathrm{L} 2$ & $\mathrm{~L} 2$ & 1.004 & 2 & \\
\hline 2M0205-1159 & L7 & L5.5 & L9 & 1.121 & 3 & \\
\hline $2 \mathrm{M} 0206+2235$ & $\ldots$ & L5.5 & L 4.5 & $\ldots$ & 5 & Blue \\
\hline $2 \mathrm{M} 0207+1355$ & L3 & L3 & $\mathrm{L} 1.5$ & $\ldots$ & $?$ & \\
\hline $2 \mathrm{M} 0208+2542$ & L1 & $\ldots$ & L1.5 & 1.054 & 4 & \\
\hline $2 \mathrm{M} 0208+2737$ & L5 & $\cdots$ & L6 & 1.063 & 3 & \\
\hline $2 \mathrm{M} 0227-1624$ & L1 & $\ldots$ & L1.5 & 1.023 & 4 & S1. red \\
\hline $2 \mathrm{M} 0228+2537$ & L0: & L0 & L0 & $\ldots$ & 4 & \\
\hline $2 \mathrm{M} 0230-3027$ & $\ldots$ & $\mathrm{L} 1$ & $\mathrm{~L} 1$ & 1.026 & 2 & \\
\hline $2 \mathrm{M} 0235-2331$ & L1 & $\mathrm{L} 1$ & L1.5 & 1.038 & 4 & \\
\hline $2 \mathrm{M} 0241-1241$ & L2: & $\ldots$ & $\mathrm{L} 2.5$ & 1.035 & 4 & \\
\hline $2 \mathrm{M} 0255-4700$ & L8 & L9 & L9 & 1.144 & 9 & \\
\hline 2M0257-3105 & L8 & $\ldots$ & L8.5 & 1.094 & 10 & \\
\hline $2 \mathrm{M} 0300-2130$ & $\ldots$ & L6 (pec) & L 4.5 & $\ldots$ & 2 & \\
\hline $2 \mathrm{M} 0310-1648$ & L8 & L9 & L9.5 & 1.140 & 1 & Sl. red \\
\hline $2 \mathrm{M} 0318-3421$ & L7 & $\ldots$ & L7 & 1.063 & 3 & \\
\hline $2 \mathrm{M} 0320-0446$ & M8 & L0.5 & L0 & $\ldots$ & 4 & Sl. blue \\
\hline $2 \mathrm{M} 0328+2302$ & L8 & L9.5 & L9.5 & 1.155 & 4 & \\
\hline $2 \mathrm{M} 0354+2540$ & L0 & L1 & L0 & $\ldots$ & 23 & \\
\hline $2 \mathrm{M} 0406-3812$ & $\mathrm{~L} 0 \gamma$ & $\mathrm{L} 1$ (pec) & $\mathrm{L} 2$ & $\ldots$ & 2 & Red? \\
\hline $2 \mathrm{M} 0439-2353$ & L5: & ... & L 4.5 & 1.067 & 1 & \\
\hline 2M0516-3332 & L3: & $\ldots$ & L6 & $\ldots$ & 3 & \\
\hline 2M0539-0059 & L5 & L5 & L5 & 1.089 & $?$ & Blue \\
\hline $2 \mathrm{M} 0624-4521$ & L5: & $\ldots$ & L6.5 & 1.055 & 10 & \\
\hline $2 \mathrm{M} 0652+4710$ & L4.5 & $\ldots$ & L5.5 & 1.038 & 3 & \\
\hline $2 \mathrm{M} 0654+6528$ & $\ldots$ & L6 & L5.5 & $\ldots$ & 3 & \\
\hline $2 \mathrm{M} 0717+5705$ & L3 & L6.5 & L3 & 1.036 & 3 & Red \\
\hline $2 \mathrm{M} 0741+0531$ & $\ldots$ & $\mathrm{L} 1.5$ & L1 & 1.023 & 2 & \\
\hline $2 \mathrm{M} 0801+4628$ & L6.5 & $\ldots$ & L5 & 1.038 & 3 & \\
\hline $2 \mathrm{M} 0805+4812$ & L4 & L9 & L5 & 1.098 & 11 & Blue \\
\hline $2 \mathrm{M} 0820+1037$ & $\ldots$ & L9.5 & L9.5 & $\ldots$ & 5 & \\
\hline $2 \mathrm{M} 0820+4500$ & L5 & $\ldots$ & L7 & 1.052 & 3 & \\
\hline $2 \mathrm{M} 0823+2428$ & L3 & $\ldots$ & $\mathrm{L} 2$ & 1.033 & 3 & S1. red \\
\hline $2 \mathrm{M} 0825+2115$ & L7.5 & L6 & L7. 5 & 1.079 & 3 & \\
\hline $2 \mathrm{M} 0828-1309$ & $\mathrm{~L} 2$ & $\ldots$ & $\mathrm{L} 1$ & 1.029 & 12 & \\
\hline $2 \mathrm{M} 0830+4828$ & L8 & L9 & L9.5 & 1.120 & 4 & \\
\hline $2 \mathrm{M} 0835+1953$ & $\ldots$ & L5 & L5 & 1.080 & 5 & \\
\hline $2 \mathrm{M} 0835-0819$ & L5 & $\ldots$ & L6.5 & 1.048 & 3 & \\
\hline $2 \mathrm{M} 0847-1532$ & $\mathrm{~L} 2$ & $\ldots$ & $\mathrm{L} 1.5$ & 1.049 & 13 & \\
\hline $2 \mathrm{M} 0850+1057$ & L6 & $\ldots$ & L6.5 & 1.059 & 14 & \\
\hline $2 \mathrm{M} 0851+1817$ & $\ldots$ & L4.5 & L6 & $\ldots$ & 5 & \\
\hline $2 \mathrm{M} 0852+4720$ & $\cdots$ & L9.5 & L9.5 & 1.128 & 3 & \\
\hline $2 \mathrm{M} 0856+2235$ & L3: & $\ldots$ & L3 & 1.033 & 3 & \\
\hline $2 \mathrm{M} 0857+5708$ & L8 & L8 & L7 & 1.097 & 3 & Red \\
\hline 2M0859-1949 & L6:: & $\ldots$ & L8 & 1.146 & 10 & \\
\hline $2 \mathrm{M} 0905+5623$ & L5 & $\ldots$ & L5 & 1.070 & 3 & \\
\hline $2 \mathrm{M} 0908+5032$ & L5 & L9 & L8 & 1.124 & 10 & Blue \\
\hline
\end{tabular}


Table 2

(Continued)

\begin{tabular}{|c|c|c|c|c|c|c|}
\hline 2MASS Name & $\begin{array}{c}\mathrm{SpT}_{\text {opt }} \\
\text { (Pub.) }\end{array}$ & $\begin{array}{c}\mathrm{SpT}_{\mathrm{NIR}} \\
\text { (Pub.) }\end{array}$ & $\begin{array}{c}\mathrm{SpT}_{\mathrm{IR}} \\
\text { (Adopted) }\end{array}$ & $\begin{array}{l}\mathrm{H}_{2} \\
(\mathrm{~K})\end{array}$ & Ref. & Note \\
\hline $2 \mathrm{M} 0915+0422$ & L7 & $\ldots$ & L6 & 1.068 & 1 & \\
\hline 2M0921-2104 & $\mathrm{L} 1.5$ & L4 & L1 & 1.043 & 12 & Blue \\
\hline $2 \mathrm{M} 0929+3429$ & L8 & L7.5 & L7 & 1.080 & 3 & \\
\hline $2 \mathrm{M} 1007+1930$ & $\ldots$ & L8 & L9 & 1.144 & 3 & \\
\hline 2M1010-0406 & L6 & L6 & L6 & 1.071 & 8 & \\
\hline $2 \mathrm{M} 1025+3212$ & $\ldots$ & L7.5 & L9 & $\ldots$ & 5 & \\
\hline $2 \mathrm{M} 1022+4114$ & L0 & L (early) & L0.5 & $\ldots$ & 4 & \\
\hline $2 \mathrm{M} 1036-3441$ & L6 & $\ldots$ & L9 & 1.126 & 3 & \\
\hline $2 \mathrm{M} 1043+1213$ & $\ldots$ & L7 & L9 & 1.156 & 3 & \\
\hline $2 \mathrm{M} 1043+2225$ & L8 & $\ldots$ & L8.5 & 1.152 & 10 & Sl. red \\
\hline $2 \mathrm{M} 1044+0429$ & $\ldots$ & L7 & L8.5 & 1.130 & 3 & \\
\hline $2 \mathrm{M} 1046+4441$ & $\ldots$ & L5 (pec) & $\mathrm{L} 4$ & 1.096 & 2 & \\
\hline $2 \mathrm{M} 1048+0111$ & L1 & L4 & $\mathrm{L} 1$ & 1.021 & 4 & \\
\hline $2 \mathrm{M} 1058-1548$ & L3 & L3 & $\mathrm{L} 2.5$ & 1.037 & 3 & \\
\hline $2 \mathrm{M} 1100+4957$ & L3.5 & $\ldots$ & L3 & 1.052 & 10 & Sl. red \\
\hline 2M1104+1959 & L4 & $\ldots$ & L4 & 1.086 & 15 & \\
\hline $2 \mathrm{M} 1112+3548$ & L4.5 & $\ldots$ & L5 & 1.042 & 3 & \\
\hline $2 \mathrm{M} 1113+3430$ & $\ldots$ & L3 & L3 & $\ldots$ & 5 & \\
\hline $2 \mathrm{M} 1121+4332$ & $\ldots$ & L7.5 & L5 & $\ldots$ & 5 & Blue \\
\hline $2 \mathrm{M} 1126-5003$ & L4.5 & L6.5 (pec) & L5 & 1.102 & 16 & Blue \\
\hline $2 \mathrm{M} 1146+2230$ & L3 & $\ldots$ & $\mathrm{L} 2.5$ & 1.041 & 3 & \\
\hline $2 \mathrm{M} 1155+0559$ & $\ldots$ & L7.5 & L5 & 1.078 & 3 & \\
\hline $2 \mathrm{M} 1207-3151$ & L3: & $\ldots$ & $\mathrm{L} 2$ & 1.036 & 3 & Red \\
\hline $2 \mathrm{M} 1216+3003$ & $\ldots$ & L3.5 & L4 & $\ldots$ & 5 & \\
\hline $2 \mathrm{M} 1219-3128$ & $\ldots$ & L8 & L9 & 1.161 & 3 & Sl. red \\
\hline $2 \mathrm{M} 1221+0257$ & L0 & $\ldots$ & L0.5 & $\ldots$ & 4 & \\
\hline $2 \mathrm{M} 1228-1547$ & L5 & L6 & L4.5 & 1.073 & 3 & \\
\hline $2 \mathrm{M} 1230+2827$ & $\ldots$ & L4: & $\mathrm{L} 2$ & 1.043 & 17 & \\
\hline $2 \mathrm{M} 1239+5515$ & L5 & $\ldots$ & L5 & 1.048 & 3 & \\
\hline $2 \mathrm{M} 1247-1117$ & $\ldots$ & L0: & L0 & $\ldots$ & 2 & \\
\hline $2 \mathrm{M} 1302-5650$ & L2 & L3 (pec) & L3 & $\ldots$ & 2 & \\
\hline $2 \mathrm{M} 1305-2541$ & L2 & L2 & L2 & 1.021 & 12 & \\
\hline $2 \mathrm{M} 1318+1736$ & $\ldots$ & $\mathrm{L} 5.5$ & L6 & 1.039 & 2 & \\
\hline $2 \mathrm{M} 1326-0038$ & L8: & L5.5 & L7 & 1.046 & 3 & \\
\hline $2 \mathrm{M} 1331+3407$ & L0 & L1 (pec) & L1 & 1.019 & 2 & S1. red \\
\hline $2 \mathrm{M} 1331-0116$ & L6 & L8 & T0 & $\ldots$ & 3 & \\
\hline $2 \mathrm{M} 1342+1340$ & $\ldots$ & L5.5 & L6 & $\ldots$ & 5 & \\
\hline $2 \mathrm{M} 1345+5216$ & $\ldots$ & $\mathrm{L} 3.5$ & L3 & $\ldots$ & 5 & \\
\hline $2 \mathrm{M} 1357+1428$ & L4: & $\ldots$ & L2 & 1.014 & 3 & \\
\hline $2 \mathrm{M} 1400+4338$ & $\ldots$ & L7 & L8 & 1.138 & 3 & \\
\hline $2 \mathrm{M} 1407+1241$ & L1:: & $\ldots$ & L4 & 1.042 & 3 & \\
\hline $2 \mathrm{M} 1416+1348$ & L6 & $\ldots$ & L5 & 1.111 & 18 & Blue \\
\hline $2 \mathrm{M} 1416+5006$ & $\ldots$ & L5.5 & L4.5 & $\ldots$ & 5 & \\
\hline $2 \mathrm{M} 1418-3538$ & $\ldots$ & $\mathrm{L} 1.5$ & $\mathrm{~L} 1.5$ & 1.030 & 2 & \\
\hline $2 \mathrm{M} 1419+5919$ & $\ldots$ & L1: & $\mathrm{L} 1$ & $\ldots$ & 2 & \\
\hline $2 \mathrm{M} 1422+2215$ & $\ldots$ & L6.5 & L5 & $\ldots$ & 5 & Blue \\
\hline $2 \mathrm{M} 1431+1436$ & L2 & L3.5 & L1 & 1.018 & 17 & Blue \\
\hline $2 \mathrm{M} 1434+2202$ & $\ldots$ & $\mathrm{L} 2.5$ & L1 & 1.045 & 17 & Blue \\
\hline $2 \mathrm{M} 1439+1929$ & $\mathrm{~L} 1$ & $\ldots$ & L1 & 1.016 & 15 & \\
\hline $2 \mathrm{M} 1440-1303$ & L1 & L1 (pec) & L1 & 1.038 & 2 & \\
\hline $2 \mathrm{M} 1448+1031$ & L4: & L3.5 & L5.5 & 1.065 & 3 & \\
\hline $2 \mathrm{M} 1506+1321$ & L3 & L3 & L3 & 1.020 & 9 & \\
\hline $2 \mathrm{M} 1515+4436$ & $\ldots$ & L7.5 & L7.5 & 1.093 & 3 & \\
\hline $2 \mathrm{M} 1523+3014$ & L8 & L8 & L8 & 1.126 & 3 & \\
\hline $2 \mathrm{M} 1526+2043$ & L7 & $\ldots$ & L5 & 1.076 & 15 & \\
\hline $2 \mathrm{M} 1534+1219$ & $\ldots$ & L4 & L3 & 1.067 & 5 & \\
\hline $2 \mathrm{M} 1540+3742$ & $\ldots$ & L9 & L9 & 1.135 & 3 & \\
\hline $2 \mathrm{M} 1548+1722$ & $\ldots$ & L5 & L4.5 & $\ldots$ & 5 & \\
\hline $2 \mathrm{M} 1550+1455$ & L2: & $\ldots$ & $\mathrm{L} 2.5$ & 1.039 & 19 & \\
\hline $2 \mathrm{M} 1617+4019$ & $\ldots$ & L4 & L6.5 & 1.064 & 5 & Sl. red \\
\hline $2 \mathrm{M} 1622+1159$ & $\ldots$ & L6 & L4.5 & $\ldots$ & 5 & \\
\hline $2 \mathrm{M} 1630+4344$ & $\ldots$ & L7 & L8.5 & 1.105 & 3 & \\
\hline $2 \mathrm{M} 1632+1904$ & L8 & L8 & L8 & 1.078 & 15 & \\
\hline $2 \mathrm{M} 1633-0640$ & $\ldots$ & L6 & L5.5 & 1.085 & 5 & Sl. blue \\
\hline $2 \mathrm{M} 1638+1327$ & $\ldots$ & L2 & L1.5 & 1.039 & 2 & \\
\hline
\end{tabular}


Table 2

(Continued)

\begin{tabular}{|c|c|c|c|c|c|c|}
\hline 2MASS Name & $\begin{array}{l}\mathrm{SpT}_{\text {opt }} \\
\text { (Pub.) }\end{array}$ & $\begin{array}{c}\text { SpT }_{\text {NIR }} \\
\text { (Pub.) }\end{array}$ & $\begin{array}{c}\mathrm{SpT}_{\mathrm{IR}} \\
\text { (Adopted) }\end{array}$ & $\begin{array}{l}\mathrm{H}_{2} \\
(\mathrm{~K})\end{array}$ & Ref. & Note \\
\hline 2M1645-1319 & $\mathrm{L} 1.5$ & $\ldots$ & $\mathrm{L} 1$ & 1.018 & 4 & \\
\hline $2 \mathrm{M} 1649+4643$ & $\ldots$ & L5 & L4.5 & $\ldots$ & 5 & Sl. blue \\
\hline $2 \mathrm{M} 1653+6231$ & L3 & $\ldots$ & $\mathrm{L} 1.5$ & 1.028 & 3 & \\
\hline 2M1705-0516 & $\ldots$ & L4 & $\mathrm{L} 1$ & 1.034 & 3 & \\
\hline $2 \mathrm{M} 1707-0558$ & $\ldots$ & L3 & $\mathrm{L} 2$ & 1.067 & 13 & \\
\hline $2 \mathrm{M} 1711+2232$ & L6.5 & $\ldots$ & L9.5 & 1.137 & 3 & \\
\hline $2 \mathrm{M} 1728+3948$ & L7 & $\ldots$ & L6.5 & 1.080 & 14 & \\
\hline $2 \mathrm{M} 1731+5310$ & $\ldots$ & L6 & L5 & 1.078 & 5 & \\
\hline $2 \mathrm{M} 1732+2656$ & $\ldots$ & L1 & L1 & 1.026 & 2 & \\
\hline $2 \mathrm{M} 1739+2454$ & $\ldots$ & L4 & $\mathrm{L} 2$ & 1.048 & 2 & \\
\hline 2M1750-0016 & $\ldots$ & $\mathrm{L} 5.5$ & L4.5 & 1.060 & 3 & Sl. blue \\
\hline $2 \mathrm{M} 1807+5015$ & $\mathrm{~L} 1.5$ & L1 & $\mathrm{L} 1$ & 1.029 & 4 & \\
\hline $2 \mathrm{M} 1813+5101$ & $\ldots$ & L5 & L4.5 & 1.094 & 2 & \\
\hline $2 \mathrm{M} 1821+1414$ & L 4.5 & L5 (pec) & L5 & 1.047 & 20 & \\
\hline 2M1916-3700 & $\ldots$ & L1 & L1 & $\ldots$ & 2 & \\
\hline 2M1928-4356 & L4 & $\ldots$ & L6 & 1.057 & 3 & \\
\hline $2 \mathrm{M} 1941+6826$ & $\ldots$ & L2 & L2 & 1.046 & 2 & \\
\hline $2 \mathrm{M} 1949+6222$ & L2 (pec) & L2 (pec) & $\mathrm{L} 2$ & $\ldots$ & 2 & \\
\hline $2 \mathrm{M} 2002-0521$ & L6 & $\ldots$ & L5.5 & 1.050 & 4 & Sl. red \\
\hline 2M2026-2943 & L1: & $\ldots$ & L1 & $\ldots$ & 21 & \\
\hline $2 \mathrm{M} 2028+0052$ & L3 & $\ldots$ & $\mathrm{L} 2.5$ & 1.048 & 15 & \\
\hline $2 \mathrm{M} 2034+0827$ & L1 & $\ldots$ & $\mathrm{L} 1$ & 1.038 & 3 & \\
\hline $2 \mathrm{M} 2036+1051$ & L3 & $\ldots$ & $\mathrm{L} 2$ & 1.041 & 3 & \\
\hline $2 \mathrm{M} 2043-1551$ & $\ldots$ & L9 & L9.5 & 1.201 & 3 & \\
\hline $2 \mathrm{M} 2057-0252$ & $\mathrm{~L} 1.5$ & $\mathrm{~L} 1.5$ & $\mathrm{~L} 1$ & 1.028 & 15 & \\
\hline $2 \mathrm{M} 2101+1756$ & L7.5 & $\ldots$ & L7.5 & 1.097 & 3 & \\
\hline 2M2104-1037 & $\mathrm{L} 2.5$ & $\ldots$ & $\mathrm{L} 2$ & 1.038 & 3 & \\
\hline 2M2130-0845 & $\mathrm{L} 1.5$ & $\ldots$ & $\mathrm{L} 1$ & 1.035 & 2 & \\
\hline 2M2131-0119 & $\ldots$ & L9 & L9.5 & $\ldots$ & 5 & \\
\hline $2 \mathrm{M} 2132+1029$ & $\ldots$ & L4.5 & $\mathrm{L} 5.5$ & $\ldots$ & 5 & \\
\hline $2 \mathrm{M} 2132+1341$ & L6 & $\ldots$ & L6 & 1.068 & 10 & \\
\hline $2 \mathrm{M} 2133+1018$ & $\ldots$ & L5 & L3.5 & $\ldots$ & 5 & \\
\hline $2 \mathrm{M} 2142-3101$ & L3 & $\ldots$ & $\mathrm{L} 2$ & 1.048 & 3 & \\
\hline $2 \mathrm{M} 2148+4003$ & L6 & L6 (pec) & L7 & $\ldots$ & 20 & Red \\
\hline $2 \mathrm{M} 2151+3402$ & $\ldots$ & L7 (pec) & L6 & 1.084 & 2 & \\
\hline $2 \mathrm{M} 2151-2441$ & L3 & $\ldots$ & L3 & 1.034 & 4 & Red \\
\hline $2 \mathrm{M} 2155+2345$ & $\ldots$ & L2 & L2 & 1.080 & 2 & \\
\hline $2 \mathrm{M} 2158-1550$ & L4: & $\mathrm{L} 4$ & $\mathrm{~L} 4$ & 1.065 & 2 & \\
\hline $2 \mathrm{M} 2211+6856$ & $\ldots$ & $\mathrm{L} 2$ & L2 & $\ldots$ & 2 & \\
\hline $2 \mathrm{M} 2212+3430$ & L5: & L6 & L6 & 1.073 & 3 & \\
\hline $2 \mathrm{M} 2249+0044$ & L3 & L5 & L7 & 1.038 & 4 & \\
\hline $2 \mathrm{M} 2252-1730$ & $\ldots$ & L7.5 & T0 & $\ldots$ & 8 & \\
\hline $2 \mathrm{M} 2317-4838$ & L4 (pec) & L6.5 (pec) & L5 & 1.046 & 2 & Red \\
\hline $2 \mathrm{M} 2328-1038$ & . & L3.5 & $\mathrm{L} 2$ & $\ldots$ & 5 & \\
\hline $2 \mathrm{M} 2351+3010$ & L5.5 & L5 (pec) & L6.5 & 1.078 & 2 & \\
\hline
\end{tabular}

References. (1) Burgasser 2007a; (2) Kirkpatrick et al. 2010; (3) Burgasser et al. 2010; (4) Burgasser et al. 2008a; (5) Chiu et al. 2006; (6) Cruz et al. 2004; (7) Dhital et al. 2011; (8) Reid et al. 2006; (9) Burgasser 2007a; (10) Siegler et al. 2007; (11) Burgasser 2007b; (12) Burgasser et al. 2007c; (13) McElwain \& Burgasser 2006; (14) Burgasser et al. 2011; (15) Burgasser et al. 2004; (16) Burgasser et al. 2008b; (17) Sheppard \& Cushing 2009; (18) Schmidt et al. 2010; (19) Burgasser et al. 2009; (20) Looper et al. 2008b; (21) Gelino \& Burgasser 2010; (22) Kirkpatrick et al. 2006; (23) Burgasser \& McElwain 2006.

\section{APPENDIX}

\section{SpeX NEAR-INFRARED SPECTRAL FITTING}

The SpeX Prism Spectral Library is an invaluable resource to the brown dwarf community. We chose to utilize this resource to extend the work of Canty et al. (2013) regarding the $\mathrm{H}_{2} \mathrm{~K}$ youth index to the entire L dwarf spectral sequence. Since many of the given or published spectral types in the Spex Prism Spectral Library were not determined homogeneously, we independently derived near-infrared spectral types for each available spectrum based on the Kirkpatrick et al. (2010) L dwarf classification scheme. For each spectrum, we first normalized to $1.28 \mu \mathrm{m}$, then performed a $\chi^{2}$ fitting to the region between 0.9 and $1.4 \mu \mathrm{m}$. Each spectrum was then inspected by-eye over the entire near-infrared range from 0.8 to $2.5 \mu \mathrm{m}$ to see which subtype was most similar to the spectrum in question, with the results of the $\chi^{2}$ fitting used as a guide. The newly derived near-infrared spectral types are given in Table 2, along with a reference to where the original near-infrared SpeX spectrum was published. We also include previously derived optical and near-infrared spectral types, when available. Those spectra with blue or red near-infrared colors compared to their best fitting spectral type are labeled as blue or red in the notes section of Table 2. The newly derived infrared spectral types of the sample 

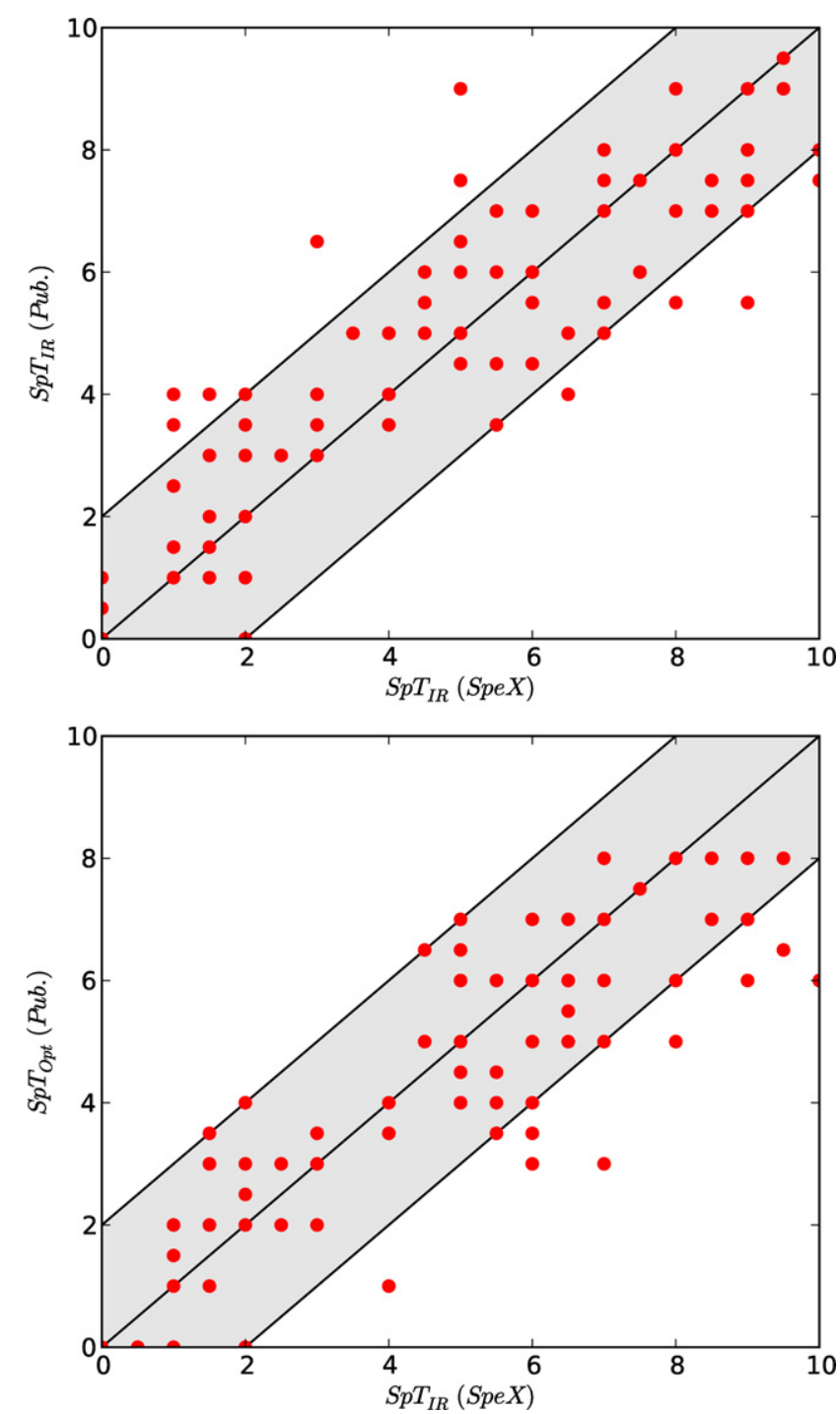

Figure 15. Left: a comparison of the spectral types derived in the Appendix and presented in Table 2 with previously published near-infrared spectral types. Any point in the shaded region agrees to within \pm 2 subtype. Right: a comparison of the spectral types derived in the Appendix and presented in Table 2 with previously published optical spectral types. We note that there are no optical spectral types defined between L8 and T0.

(A color version of this figure is available in the online journal.)

agree to within \pm 2 subtypes at a rate of $\sim 89 \%(101 / 114)$ with previously published infrared spectral types and $\sim 93 \%$ $(103 / 111)$ with optical spectral types. A comparison of the newly derived spectral types with previously published spectral types is shown in Figure 15.

\section{REFERENCES}

Allard, F., Homeier, D., \& Freytag, B. 2011, in ASP Conf. Ser. 448, 16th Cambridge Workshop on Cool Stars, Stellar Systems, and the Sun, ed. C. Johns-Krull, M. K. Browning, \& A. A. West (San Francisco, CA: ASP), 91 Allers, K. N., \& Liu, M. C. 2013, ApJ, 772, 79

Asplund, M., Grevesse, N., Sauval, A. J., \& Scott, P. 2009, ARA\&A, 47, 481 Baraffe, I., Chabrier, G., Barman, T. S., Allard, F., \& Hauschildt, P. H. 2003, A\&A, 402, 701

Barman, T., Macintosh, B., Konopacky, Q. M., \& Marois, C. 2011, ApJ, 733, 65

Bonnefoy, M., Chauvin, G., Lagrange, A.-M., et al. 2013, arXiv:1306.3709 Bowler, B. P., Liu, M. C., \& Cushing, M. 2009, ApJ, 706, 1114

Burgasser, A. J. 2007a, ApJ, 659, 655

Burgasser, A. J. 2007b, AJ, 134, 1330

Burgasser, A. J., Bardalez-Gagliuffi, D. C., \& Gizis, J. E. 2011, AJ, 141, 70
Burgasser, A. J., Cruz, K. L., Cushing, M. C., et al. 2010, ApJ, 710, 1142

Burgasser, A. J., Dhital, S., \& West, A. A. 2009, AJ, 138, 1563

Burgasser, A. J., Geballe, T. R., Leggett, S. K., Kirkpatrick, J. D., \& Golimowski, D. A. 2006, ApJ, 637, 1067

Burgasser, A. J., Liu, M. C., Ireland, M. J., Cruz, K. L., \& Dupuy, T. J. 2008a, ApJ, 681, 579

Burgasser, A. J., Looper, D. L., Kirkpatrick, J. D., Cruz, K. L., \& Swift, B. J. 2008b, ApJ, 674, 451

Burgasser, A. J., Looper, D. L., Kirkpatrick, J. D., \& Liu, M. C. 2007c, ApJ, 658,557

Burgasser, A. J., \& McElwain, M. W. 2006, AJ, 131, 1007

Burgasser, A. J., McElwain, M. W., Kirkpatrick, J. D., et al. 2004, AJ, 127, 2856

Canty, J., Lucas, P., Roche, P., \& Pinfield, D. 2013, MNRAS, 435, 2650

Chabrier, G., Baraffe, I., Allard, F., \& Hauschildt, P. 2000, ApJ, 542, 464

Chiu, K., Fan, X., Leggett, S. K., et al. 2006, AJ, 131, 2722

Cruz, K. L., Burgasser, A. J., Reid, I. N., \& Liebert, J. 2004, ApJL, 604, L61

Cruz, K. L., Kirkpatrick, J. D., \& Burgasser, A. J. 2009, AJ, 137, 3345

Cruz, K. L., Reid, I. N., Kirkpatrick, J. D., et al. 2007, AJ, 133, 439

Cushing, M. C., Marley, M. S., Saumon, D., et al. 2008, ApJ, 678, 1372

Cushing, M. C., Rayner, J. T., \& Vacca, W. D. 2005, ApJ, 632, 1115

Cushing, M. C., Saumon, D., \& Marley, M. S. 2010, AJ, 140, 1428

Cushing, M. C., Vacca, W. D., \& Rayner, J. T. 2004, PASP, 116, 362

Cutri, R. M., Skrutskie, M. F., van Dyk, S., et al. 2003, yCat, 2246, 0

Cutri, R. M., et al. 2012, yCat, 2311, 0

Dahn, C. C., Harris, H. C., Vrba, F. J., et al. 2002, AJ, 124, 1170

Dhital, S., Burgasser, A. J., Looper, D. L., \& Stassun, K. G. 2011, AJ, 141, 7

Dupuy, T. J., \& Liu, M. C. 2012, ApJS, 201, 19

Faherty, J. K., Burgasser, A. J., Cruz, K. L., et al. 2009, AJ, 137, 1

Faherty, J. K., Burgasser, A. J., Walter, F. M., et al. 2012, ApJ, 752, 56

Faherty, J. K., Rice, E., Cruz, K. L., et al. 2013, AJ, 145, 2

Geballe, T. R., Knapp, G. R., Leggett, S. K., et al. 2002, ApJ, 564, 466

Gelino, C. R., \& Burgasser, A. J. 2010, AJ, 140, 110

Gizis, J. E., Faherty, J. K., Liu, M., et al. 2012, AJ, 144, 94

Golimowski, D. A., Leggett, S. K., Marley, M. S., et al. 2004, AJ, 127, 3516

Horne, K. 1986, PASP, 98, 609

Kirkpatrick, J. D., Barman, T. S., Burgasser, A. J., et al. 2006, ApJ, 639, 1120

Kirkpatrick, J. D., Cushing, M. C., Gelino, C. R., et al. 2011, ApJS, 197, 19

Kirkpatrick, J. D., Cruz, K. L., Barman, T., et al. 2008, ApJ, 689, 1295

Kirkpatrick, J. D., Looper, D. L., Burgasser, A. J., et al. 2010, ApJS, 190, 100

Liu, M. C., Dupuy, T. J., \& Allers, K. N. 2013, AN, 334, 85

Liu, M. C., Magnier, E. A., Deacon, N. R., et al. 2013, ApJL, 777, L20

Looper, D. L., Burgasser, A. J., Gelino, C. R., \& Kirkpatrick, J. D. 2008a, ApJ, 685,1183

Looper, D. L., Kirkpatrick, J. D., \& Burgasser, A. J. 2007, AJ, 134, 1162

Looper, D. L., Kirkpatrick, J. D., Cutri, R. M., et al. 2008b, ApJ, 686, 528

Lucas, P. W., Roche, P. F., Allard, F., \& Hauschildt, P. H. 2001, MNRAS, 326, 695

Mace, G. N., Kirkpatrick, J. D., Cushing, M. C., et al. 2013, ApJS, 205, 6

Malo, L., Doyon, R., Lafreniere, D., et al. 2013, ApJ, 762, 88

Marley, M. S., Saumon, D., Cushing, M., et al. 2012, ApJ, 754, 135

McElwain, M. W., \& Burgasser, A. J. 2006, AJ, 132, 2074

McLean, I. S., McGovern, M. R., Burgasser, A. J., et al. 2003, ApJ, 596, 561

McLean, I. S., Prato, L., McGovern, M. R., et al. 2007, ApJ, 658, 1217

Oppenheimer, B. R., Baranec, C., Beichman, C., et al. 2013, ApJ, 768, 24

Rayner, J. T., Cushing, M. C., \& Vacca, W. D. 2009, ApJS, 185, 289

Rayner, J. T., Toomey, D. W., Onaka, P. M., et al. 2003, PASP, 115, 362

Reid, I. N., Lewitus, E., Burgasser, A. J., \& Cruz, K. L. 2006, ApJ, 639, 1114

Rice, E. L., Faherty, J. K., Cruz, K., et al. 2011, in ASP Conf. Ser. 448, 16th Cambridge Workshop on Cool Stars, Stellar Systems, and the Sun, ed. C. Johns-Krull (San Francisco, CA: ASP), 187

Rodriguez, D., Zuckerman, B., Kastner, J. H., et al. 2013, ApJ, 774, 101

Saumon, D., \& Marley, M. S. 2008, ApJ, 689, 1327

Schmidt, S. J., Cruz, K. L., Bongiorno, B. J., Liebert, J., \& Reid, I. N. 2007, AJ, 133,2258

Schmidt, S. J., West, A. A., Burgasser, A. J., Bochanski, J. J., \& Hawley, S. L. 2010, AJ, 139, 1045

Schneider, A. C. 2013, PhD thesis, Univ. Georgia

Sheppard, S. S., \& Cushing, M. C. 2009, AJ, 137, 304

Siegler, N., Close, L. M., Burgasser, A. J., et al. 2007, AJ, 133, 2320

Simcoe, R. A., Burgasser, A. J., Schechter, P. L., et al. 2013, PASP, 125, 270

Thompson, M., Kirkpatrick, J. D., \& Mace, G. N. 2013, PASP, 125, 809

Torres, C. A. O., Quast, G. R., Melo, C. H. F., \& Sterzik, M. F. 2008, in Handbook of Star Forming Regions, Vol. II: The Southern Sky, ed. B. Reipurth (ASP Monograph Publications, Vol. 5; San Francisco, CA: ASP), 757

Vacca, W. D., Cushing, M. C., \& Rayner, J. T. 2003, PASP, 115, 389

Wright, E. L., Eisenhardt, P., Mainzer, A., et al. 2010, AJ, 140, 1868

Zuckerman, B., \& Song, I. 2004, ARA\&A, 42, 685 